\title{
New signposts of massive star formation in the S235A-B region
}

\author{
M. Felli ${ }^{1}$, F. Massi ${ }^{1}$, M. Robberto ${ }^{2}$, and R. Cesaroni ${ }^{1}$ \\ 1 INAF-Osservatorio Astrofisico di Arcetri, Largo E. Fermi 5, 50125 Firenze, Italy \\ e-mail: fmassi@arcetri.astro.it \\ 2 Space Telescope Science Institute, 3700 San Martin Drive, Baltimore, MD 21218, USA \\ Received 5 December 2005 / Accepted 15 February 2006
}

\begin{abstract}
Aims. We report on new aspects of the star-forming region S235AB revealed through high-resolution observations at radio and midinfrared wavelengths.

Methods. Using the Very Large Array, we carried out sensitive observations of S235AB in the cm continuum $(6,3.6,1.3$, and 0.7$)$ and in the $22 \mathrm{GHz}$ water maser line. These were complemented with Spitzer Space Telescope Infrared Array Camera archive data to clarify the correspondence between radio and IR sources. We made also use of newly presented data from the Medicina water maser patrol, started in 1987, to study the variability of the water masers found in the region.

Results. S235A is a classical HII region whose structure is now well resolved. To the south, no radio continuum emission is detected either from the compact molecular core or from the jet-like structure observed at $3.3 \mathrm{~mm}$, suggesting emission from dust in both cases. We find two new compact radio continuum sources (VLA-1 and VLA-2) and three separate maser spots. VLA-1 coincides with one of the maser spots and with a previously identified IR source (M1). VLA-2 lies towards S235B and represents the first radio detection from this peculiar nebula that may represent an ionized wind from a more evolved star. The two other maser spots coincide with an elongated structure previously observed within the molecular core in the $\mathrm{C}^{34} \mathrm{~S}$ line. This structure is perpendicular to a bipolar molecular outflow observed in $\mathrm{HCO}^{+}(1-0)$ and may trace the associated equatorial disk. The Spitzer images reveal a red object towards the molecular core. This is the most viable candidate for the embedded source originating the outflow and maser phenomenology.

Conclusions. The picture emerging from these and previous data shows the extreme complexity of a small $(\leq 0.5 \mathrm{pc})$ star-forming region where widely different stages of stellar evolution are present.
\end{abstract}

Key words. stars: formation - ISM: individual objects: S235A-B - ISM: jets and outflows - radio continuum: ISM - masers

\section{Introduction}

This paper continues the study of the star-forming complex S235A-B (see Felli et al. 2004, and references therein), focussing in particular on a deeply embedded Young Stellar Object (YSO) found between S235A and S235B, close to a water maser. The presence of the YSO is implied from the typical signposts of early stellar evolution, including two molecular outflows, a hot molecular core, a sub-millimeter peak, and a water maser. The YSO represents the youngest object in the star-forming complex.

The site morphology derived from all previous observations is summarised in Fig. 1. S235A is a small optical nebulosity coinciding with a compact, but well-resolved, HII region. It appears as a less-evolved region, with respect to the more extended and diffuse HII region S235 located further north, and probably is unrelated to the S235A-B complex. S235A lies at the northern edge of a molecular clump that represents the brightest peak of a more extended molecular cloud (Evans \& Blair 1981; Nakano \& Yoshida 1986; Cesaroni et al. 1999).

Lying $\sim 40^{\prime \prime}$ south of S235A (0.35 pc at the assumed distance of $1.8 \mathrm{kpc}$, Nakano \& Yoshida 1986), at the SW edge of the molecular core, S235B is a smaller diffuse nebulosity detected both in the optical and near-IR, exhibiting a near-IR excess and intense emission in optical and IR hydrogen lines $(\mathrm{H} \alpha, \mathrm{Br} \gamma)$, but without a radio continuum counterpart, making it a rather peculiar object. In $\mathrm{H} \alpha$, it consists of an unresolved peak superimposed on a circular nebula that is $\sim 10^{\prime \prime}$ in diameter
(Krassner et al. 1982; Alvarez et al. 2004). S235B appears to be a young star with an expanding ionized envelope surrounded by a diffuse nebulosity (Felli et al. 1997).

A large-scale molecular outflow was first found in ${ }^{12} \mathrm{CO}(1-0)$ by Nakano \& Yoshida (1986) with a resolution of $\sim 14^{\prime \prime}$; it was centred at S235B and aligned in a NE-SW direction, about $35^{\prime \prime}(0.3 \mathrm{pc})$ in length. Felli et al. (1997) confirmed the blue lobe of the outflow in ${ }^{13} \mathrm{CO}(2-1)$ with a resolution of $\sim 11^{\prime \prime}$, but failed to detect the red lobe.

Near-IR images revealed a highly obscured stellar cluster between S235A and S235B, i.e. centred on the water maser, with several sources with IR excess (Felli et al. 1997), in particular source M 1, which exhibits the largest near-IR excess. This source has been suggested to be the candidate YSO supplying energy to the $-60 \mathrm{~km} \mathrm{~s}^{-1}$ water maser, but with great uncertainty since it lies more than $5^{\prime \prime}$ to the south (see also Fig. 1).

The above picture was clarified by Felli et al. (2004), who presented high-resolution (between $2^{\prime \prime}$ and $\left.4^{\prime \prime}\right) \mathrm{mm}$ line $\left(\mathrm{HCO}^{+}\right.$, $\mathrm{C}^{34} \mathrm{~S}, \mathrm{H}_{2} \mathrm{CS}, \mathrm{SO}_{2}$, and $\left.\mathrm{CH}_{3} \mathrm{CN}\right)$ and continuum observations, together with far-IR observations.

A compact molecular core (hereafter, the mm core) was found both in the $\mathrm{mm}$ continuum (hot dust emission, $T_{\text {dust }}=$ $T_{\mathrm{CH}_{3} \mathrm{CN}} \sim 30 \mathrm{~K}$ ) and in the molecular lines, peaking close to the water maser position and well-separated from S235A and S235B. Two molecular outflows were found in $\mathrm{HCO}^{+}(1-0)$ centred on the $\mathrm{mm}$ core. One of them (hereafter, the NE-SW outflow) is aligned along the same NE-SW direction of the 


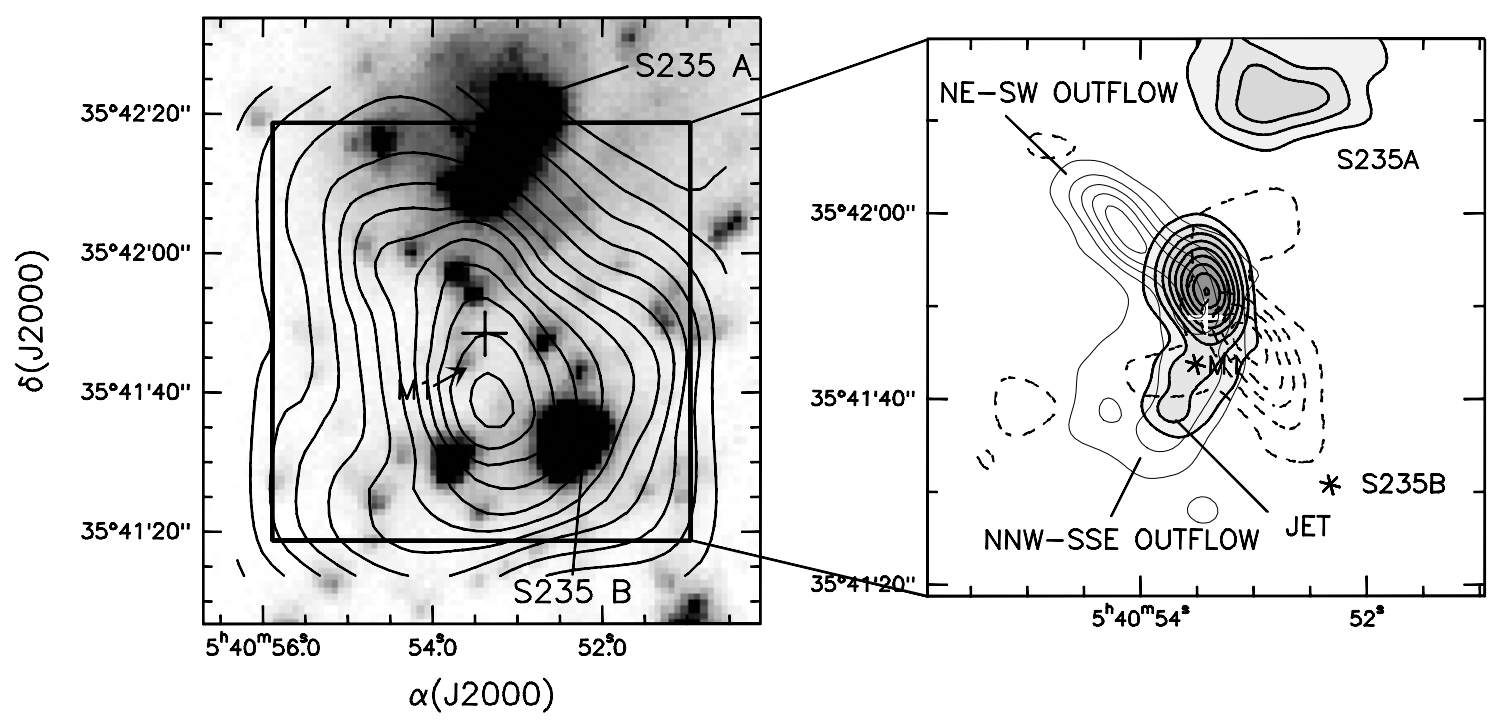

Fig. 1. Overview of the S235A-B star forming complex Left: overlay of the single-dish HCN map (contours) from Cesaroni et al. (1999) with the $K$-band image of the S235A-B region from Felli et al. (1997). Right: overlay of the interferometric maps of the 3.3 mm continuum emission (thick contours and grey scale) with the $\mathrm{HCO}^{+}(1-0)$ outflows (thin solid line: $V<-20.9 \mathrm{~km} \mathrm{~s}^{-1}$, dashed line: $V>-15.5 \mathrm{~km} \mathrm{~s}^{-1}$ ) from Felli et al. (2004). In both, a cross marks the location of the $\mathrm{H}_{2} \mathrm{O}$ maser at $-61.2 \mathrm{~km} \mathrm{~s}^{-1}$ from Tofani et al. (1995). The infrared source with the largest near-IR excess detected in previous works, M 1, is also indicated. The offsets between M 1, the water maser, the HCN peak, and the mm continuum peak are all larger than the position uncertainties.

large-scale outflow detected by Nakano \& Yoshida (1986). It spans $\sim 0.4 \mathrm{pc}$, and has an estimated mass of $9 M_{\odot}$ and a mechanical luminosity $>19 L_{\odot}$. The other (hereafter, the NNW-SSE outflow) is more compact and aligned in a NNW-SSE direction. It spans $\sim 0.3 \mathrm{pc}$ and has an estimated mass of $4 M_{\odot}$ and a mechanical luminosity of $\sim 0.3 L_{\odot}$.

Felli et al. (2004) derived an upper limit of $\sim 10^{3} L_{\odot}$ for the bolometric luminosity of the $\mathrm{mm}$ core and suggested the presence of an embedded intermediate-mass YSO driving the NE-SW outflow and supplying the energy for the $-60 \mathrm{~km} \mathrm{~s}^{-1}$ water maser. Studying the high velocity red and blue emission of $\mathrm{C}^{34} \mathrm{~S}(5-4)$ towards the mm core, they also found a compact structure with a velocity gradient perpendicular to the NE-SW outflow that might represent the signature of a circumstellar disk.

An elongated structure (called a "jet") protruding from the mm core and coinciding with the blue lobe of the NNW-SSE outflow was also detected in the continuum at $3.3 \mathrm{~mm}$ (see Fig. 1, right) and, with a lower signal-to-noise ratio, also at $1.2 \mathrm{~mm}$. The spectral index of the jet, $\alpha \sim 0.6$ (defined as $S_{v} \propto v^{\alpha}$ ) is rather uncertain, but different from that of the mm core, $\alpha \sim 2.5$, suggesting that the emission might arise in an ionized wind rather than being due to dust. Radio jets are often observed at the base of molecular outflows (Rodríguez 1997; Anglada et al. 1998; Beltrán et al. 2001) in both low-mass and high-mass starforming regions (e.g., Rodríguez 1996). They are characterized by spectral indices in the range -0.1 to $\sim 1$ and are elongated in the outflow direction. Expanding ionized envelopes also have a spectral index $\alpha=0.6$ (Panagia \& Felli 1975).

Water masers are one of the most reliable signposts of early phases in star formation (see e.g. Tofani et al. 1995) since they provide the best indication of the position of the required powering source, i.e. the YSO. They occur both in low-mass (see, e.g., Furuya et al. 2001, 2003) and in high-mass (see Churchwell 2002, and references therein) star-forming regions and are often found to be associated with outflowing matter. Sometimes they are also found in close association with radio jets (e.g. Gómez et al. 1995).
The presence of a water maser in this region had been known since the observations of Henkel et al. (1986) and Comoretto et al. (1990), but with insufficient spatial resolution to properly locate it in the region. Only with Very Large Array (VLA) cm line observations (Tofani et al. 1995) and interferometric mm observations of the continuum sources (Felli et al. 2004), was the location of the water maser in-between S235A and S235B, almost coincident with the $\mathrm{mm}$ core, firmly established. This proved that the water maser is not associated with either of the two nebulosities and that a local early type star, presumably the YSO within the $\mathrm{mm}$ core, is needed for its excitation. In the VLA observations, maser emission was only searched for in a limited velocity range, around $-60 \mathrm{~km} \mathrm{~s}^{-1}$, since at the time this was the only component detected by single-dish observations.

The water maser in S235A-B has been monitored with the Medicina radio telescope since 1987, with coverage $\sim 4$ times per year since 1993. These observations revealed at least three separate velocity components: the one already known at $\sim-60 \mathrm{~km} \mathrm{~s}^{-1}$, one between -20 to $-30 \mathrm{~km} \mathrm{~s}^{-1}$, and one between -10 to $10 \mathrm{~km} \mathrm{~s}^{-1}$. The last two are always very weak ( $\max 10-20 \mathrm{Jy})$ and exhibit strong variations. Whether they are all related to the same YSO or to separate ones could not be established from single-dish observations because of low spatial resolution. This drove us to carry out new VLA line observations at $22 \mathrm{GHz}$ covering the whole maser velocity interval. All three velocity components found in the Medicina data were active at the time of this VLA observation.

The S235A-B region contains other masers, namely methanol $\left(\mathrm{CH}_{3} \mathrm{OH}\right)$ and $\mathrm{SiO}$ (Nakano \& Yoshida 1986; Haschick et al. 1990; Harju et al. 1998). Kurtz et al. (2004) included S235A-B in their recent VLA survey of the $\mathrm{CH}_{3} \mathrm{OH}$ maser line at $44 \mathrm{GHz}$. This is a class I methanol source, and it is believed to trace outflow activity. These authors found a cluster of $6 \mathrm{CH}_{3} \mathrm{OH}$ masers spread over an area of a few square arcsec around the water maser spot at $\sim-60 \mathrm{~km} \mathrm{~s}^{-1}$.

To clarify the nature of the embedded YSO and its relation with the outflow found in the S235A-B region, we have 
Table 1. Summary of VLA observations.

\begin{tabular}{|c|c|c|c|c|c|c|c|}
\hline \multirow[t]{2}{*}{ Date } & \multirow{2}{*}{$\begin{array}{c}\text { Frequency } \\
(\mathrm{GHz})\end{array}$} & \multicolumn{2}{|c|}{ Synthesized beam ${ }^{a}$} & \multirow{2}{*}{$\begin{array}{c}\text { Largest } \\
\text { angular scale } \\
(\operatorname{arcsec})\end{array}$} & \multirow{2}{*}{$\begin{array}{c}\text { Int. time } \\
\text { (s) }\end{array}$} & \multirow{2}{*}{$\begin{array}{c}\mathrm{rms} \\
(\mathrm{mJy} / \text { beam })\end{array}$} & \multirow[t]{2}{*}{ Notes } \\
\hline & & $\begin{array}{c}\text { Size } \\
(\operatorname{arcsec} \times \operatorname{arcsec})\end{array}$ & $\begin{array}{c}\text { PA } \\
\text { (degree) }\end{array}$ & & & & \\
\hline $07 / 03 / 2004,26 / 02 / 2004$ & 4.75 & $4.8 \times 4.3$ & -8.2 & 300 & 1200 & 0.09 & \\
\hline 07/03/2004 & 8.45 & $2.8 \times 2.6$ & -20.8 & 180 & 1800 & 0.08 & \\
\hline $26 / 02 / 2004$ & 23 & $1 \times 1$ & -40 & 60 & 7224 & 0.03 & cont. \\
\hline $07 / 03 / 2004$ & 23 & $1 \times 0.9$ & -83.7 & 60 & 434 & 0.05 & line \\
\hline 07/03/2004 & 45 & $0.6 \times 0.6$ & 88.6 & 43 & $17590^{b}$ & 0.08 & \\
\hline
\end{tabular}

${ }^{a}$ Natural weighting. ${ }^{b}$ Fast switching.

performed an extensive observational program using the VLA and the Medicina radio telescopes, complemented by archival Spitzer data.

The two primary goals of the new VLA continuum observations were: 1) to clarify the nature of the "jet" and to derive its spectrum over a larger frequency interval and 2) to search further for $\mathrm{cm}$ emission from ionized hydrogen in the $\mathrm{mm}$ core. VLA observations in the water maser line together with the Medicina patrol can give indications on the location and activity of the maser in the star-forming region. Finally, for a better understanding of the precise correspondence between IR and radio sources, in particular the precise role of M 1 and the possibility of detecting IR emission from the $\mathrm{mm}$ core, archive SpitzerIRAC observations of the S235A-B region in the four wavelengths $(3.6,4.5,5.8$, and $8 \mu \mathrm{m})$ were retrieved and analyzed.

In Sect. 2 we describe the observations, and in Sect. 3 we present the results, while in Sect. 4 we discuss our findings and how they enrich our current understanding of the S235A-B region. In Sect. 5, the main results are summarised. The reader can refer to Fig. 16 for a comprehensive sketch of the S235A-B star forming-region, including the latest data.

\section{Observations and data reduction}

\subsection{VLA observations}

The observations carried out with the VLA of the National Radio Astronomy Observatory (NRAO) ${ }^{1}$ were made with the VLA in the $\mathrm{C}$ configuration on February 26 and on March 7, 2004. The location of the water maser at $-60 \mathrm{~km} \mathrm{~s}^{-1}$ was used as phase centre; its coordinates are:

$\alpha(2000)=05^{\mathrm{h}} 40^{\mathrm{m}} 53.42^{\mathrm{s}}, \quad \delta(2000)=35^{\circ} 41^{\prime} 48^{\prime \prime} .8$.

The observations consisted of calibrator-target-calibrator scans; in the $Q$-band $(0.7 \mathrm{~cm})$ the fast-switching mode was used. The phase calibrator was $0555+398$, with boot-strapped fluxes of $5.3 \mathrm{Jy}(6 \mathrm{~cm}), 4.55 \mathrm{Jy}(3.6 \mathrm{~cm}), 2.71 \mathrm{Jy}(1.3 \mathrm{~cm})$, and $1.85 \mathrm{Jy}(0.7 \mathrm{~cm})$; the absolute amplitude calibrator was $3 \mathrm{C} 147$ $(0542+498)$, with assumed fluxes of $7.9 \mathrm{Jy}(6 \mathrm{~cm}), 4.8 \mathrm{Jy}$ $(3.6 \mathrm{~cm}), 1.8 \mathrm{Jy}(1.3 \mathrm{~cm})$, and $0.9 \mathrm{Jy}(0.7 \mathrm{~cm})$. The pointing accuracy was checked on $0555+398$ (at $3.6 \mathrm{~cm}$ ) every hour. Line observations at $1.3 \mathrm{~cm}$ were carried out with the correlator set to 64 channels and a bandwidth of $6.25 \mathrm{MHz}$. This resulted in a velocity resolution of $1.3 \mathrm{~km} \mathrm{~s}^{-1}$ over a range of $84 \mathrm{~km} \mathrm{~s}^{-1}$, sufficient to cover the whole velocity range of the water masers. The receivers were tuned so as to have the band centred at $-30 \mathrm{~km} \mathrm{~s}^{-1}$ with respect to the Local Standard of Rest. A summary of the observations is contained in Table 1 .

1 The National Radio Astronomy Observatory is a facility of the National Science Foundation operated under cooperative agreement by Associated Universities, Inc.
Since the forthcoming analysis is based on a comparison of the position of the radio sources with those of near- and mid-IR sources, we checked whether the radio coordinate system is consistent with the near-IR coordinate system. To this end, we searched the 2MASS point source catalogue for a near-IR counterpart of our phase calibrator, finding an object at the same position as the calibrator within $0 . ' 1$. This gives us confidence that the two coordinate systems are consistent with each other within this limit.

\subsection{Medicina observations}

The single-dish Medicina radio telescope ${ }^{2}$ (HPBW 1'.9) observations are part of a monitoring project of a large sample of water masers. An autocorrelator with 1024 channels and a $10 \mathrm{MHz}$ bandwidth is usually employed. The typical sensitivity for a 5-min integration is of the order of $1 \mathrm{Jy}$, and the average calibration error is about $20 \%$. For a more detailed description of the radio telescope and the relevant parameters of the water masers patrolling, we refer to Valdettaro et al. (2002) and Brand et al. (2003).

\subsection{Spitzer-IRAC observations}

Spitzer-IRAC observations of a large area around the S235A-B region in the four wavelengths $(3.6,4.5,5.8$, and $8 \mu \mathrm{m})$ were extracted from the Spitzer public archive. The observations are part of the GTO program 201 "The Role of Photodissociation Regions in High Mass Star Formation" (Principal Investigator G. Fazio). Integration time is $12 \mathrm{~s}$ at all filters.

The positional accuracy is better than $1^{\prime \prime}$. Point-source FWHM resolutions range from $\sim 1^{\prime \prime} .6$ at $3.6 \mu \mathrm{m}$ to $\sim 1^{\prime \prime} .9$ at $8.0 \mu \mathrm{m}$. The IRAC bands are large and may contain various features, depending on the environment being observed. Among these, the most important for our case are polycyclic aromatic hydrocarbon (PAH) features (3.6, 5.8, and 8.0 bands) and the $\operatorname{Br} \alpha$ line ( 4.5 band). An overview of IRAC is given by Fazio et al. (2004a).

\section{Results}

\subsection{Spitzer-IRAC observations}

A colour coded image of the region covering S235A-B, obtained by combining $4.5,5.8$ and $8.0 \mu \mathrm{m}$ observations, is shown in Fig. 2.

The two diffuse nebulosities S235A and S235B clearly dominate the extended emission.

\footnotetext{
${ }^{2}$ The Medicina VLBI radio telescope is operated by the Radioastronomy Institute of INAF, Italy.
} 


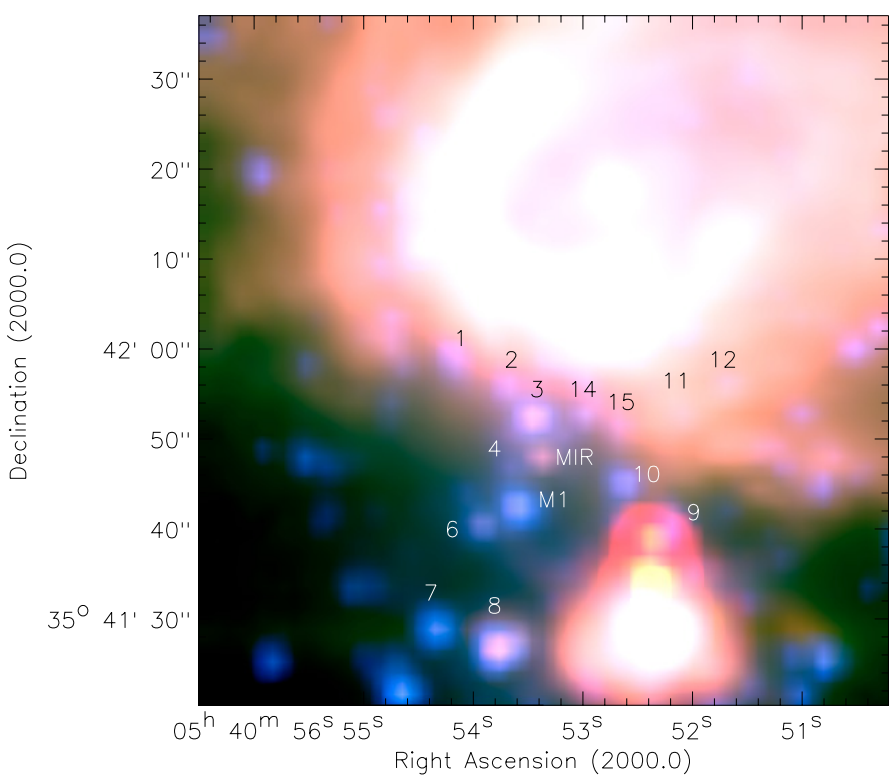

Fig. 2. Three colour (4.5 blue, 5.8 green, and $8.0 \mu \mathrm{m}$ red) image of the S235A-B region. The labels indicate the position of M 1, S235AB-MIR, and the other stars of the cluster for which we performed photometry. (A colour version of this figure is available in the on-line edition.)

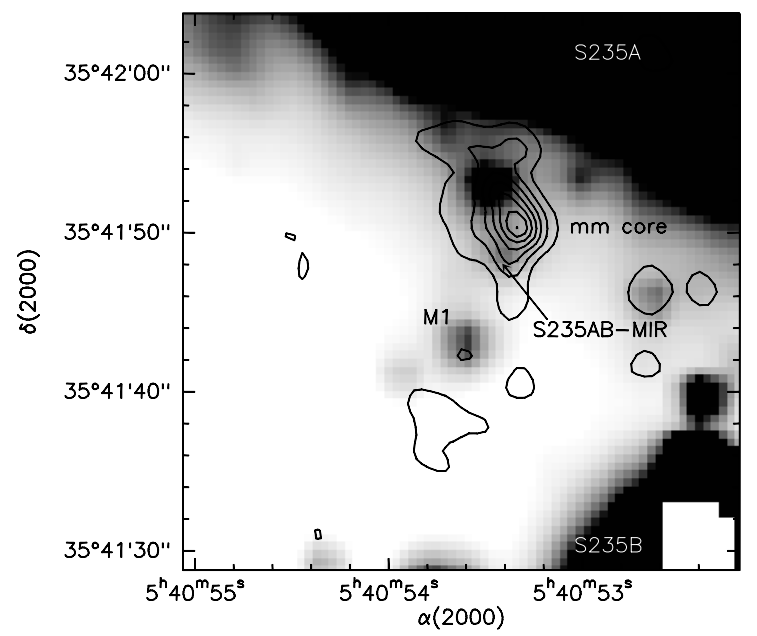

Fig. 3. The $1.2 \mathrm{~mm}$ core (contours) from Felli et al. (2004) overlaid on the $5.8 \mu \mathrm{m}$ Spitzer-IRAC image (grey scale, S235A and S235B have been saturated to show the weak emission from S235AB-MIR). The positions of the mm core, S235AB-MIR, and M 1 are indicated.

Most of the point sources detected in the $K$-band and shown in Fig. 1 are also present here. Two sources deserve more attention in view of the present work: 1) M 1, which is detected at all bands; and 2) a source (hereafter, S235AB-MIR) detected only at $4.5,5.8$, and $8.0 \mu \mathrm{m}$, which is present in the area of the mm core. Both sources are marked in Figs. 2 and 3 where we show an overlay of the $\mathrm{mm}$ core (at $1.2 \mathrm{~mm}$ ) with the $5.8 \mu \mathrm{m}$ IRAC image. S235AB-MIR lies 1'. 5 to the south of the peak of the $\mathrm{mm}$ core.

We performed aperture photometry on the IRAC sources found near to the mm-core by using DAOPHOT in IRAF. For all four bands, we selected a radius of 2 pix $(\sim 1$ FWHM; 1 pix $\sim 11^{\prime \prime} 2$ ) and a 2-pix wide annulus with an inner radius of 4 pix, to account for the highly variable background. We applied aperture corrections as estimated from the IRAC PSFs retrieved from the Spitzer Web Page (http://ssc.spitzer.caltech.edu/obs/). To derive the photometric zero points, we used the zero-magnitude fluxes given by Fazio et al. (2004b).

Photometry was done only on the most relevant sources present in the area of the S235A-B cluster, which are indicated in Fig. 2. Positions and flux densities in the four IRAC bands of the sources labelled in Fig. 2 are given in Table 2

Colour-colour plots are shown in Fig. 4. In the [5.8]-[8.0]/[3.6]-[4.5] plot, the region occupied by Class I and Class II objects (Allen et al. 2004) is indicated. M 1 has colours typical of a Class I object, while S235AB-MIR is located in a part of the plot redward of that occupied by YSOs of mass $\sim 6 M_{\odot}$, according to Whitney et al. (2004), i.e. the region corresponding to $[5.8]-[8.0] \geq 1$ and $[3.6]-[4.5] \geq 1$.

\subsection{Radio continuum}

\subsubsection{S235A}

The VLA radio continuum maps are dominated by the emission from the compact HII region S235A. We calculated the integrated flux at all frequencies over the area within the $3 \sigma$ contour of the emission at $6 \mathrm{~cm}$ (i.e. at the lowest resolution); the obtained values are listed in Table 3 . The integrated flux at $6 \mathrm{~cm}$ is in very good agreement with previous measurements, yielding $270 \pm 40 \mathrm{mJy}$. Israel \& Felli (1978) found $220 \pm 30 \mathrm{mJy}$ at $21 \mathrm{~cm}$ and suggested a partially thick emission at $6 \mathrm{~cm}$, but the ratio of fluxes at 6 and $3.6 \mathrm{~cm}$ that we derive from our data is in agreement with an optically thin emission. The presence of an ionizing star of spectral type B0.5 derived from the radio fluxes in previous works (e.g. Felli et al. 1997) is therefore confirmed. In Fig. 5, we show the maps of S235A at 6, 3.6, and $1.3 \mathrm{~cm}$. The $3.6 \mathrm{~cm}$ map is overlaid on the $5.8 \mu \mathrm{m}$ Spitzer-IRAC image and shows a well-resolved spherical shape. The Spitzer-IRAC image reveals the ionizing star S235A* at the centre of the nebula, previously detected in the $K$ band (Felli et al. 1997). The main feature of the radio maps is the asymmetry of the isophotal contours in the SE-NW direction. In the IRAC images, the morphology clearly indicates the presence of a brighter ridge SE of $\mathrm{S} 235 \mathrm{~A}^{*}$.

The contours at $1.3 \mathrm{~cm}$ outline the brightest parts of the radio ridge. In the original maps, the emission is more fragmented because of the high resolution and low surface brightness. In Fig. 5, the map has been smoothed to a resolution of $3^{\prime \prime}$. At 1.3 and $0.7 \mathrm{~cm}$, the radio fluxes are lower than expected from an optically thin emission, as was also found by Felli et al. (2004) at $3.3 \mathrm{~mm}$. We attribute this to an instrumental effect caused by the filtering of extended structures in the interferometric observations (see Table 1).

\subsubsection{The $\mathrm{mm}$ core, the jet, and the radio compact sources VLA- 1 and VLA-2}

At none of the 4 VLA wavelengths were we able to detect emission from the $\mathrm{mm}$ core where the presence of an intermediatemass YSO is suggested by the mm observations. This excludes any thermal emission from a UCHII region associated with the YSO with a flux density above the noise level given in Table 1 and implies that emission from the core is dominated by dust. At the same time, our non-detection is not in contradiction with the existence of a dust core. In fact, extrapolating at $0.7 \mathrm{~cm}$, the flux density measured at $3.3 \mathrm{~mm}(20 \mathrm{mJy})$ with a spectral index of 2.5 (Felli et al. 2004), we obtain $3 \mathrm{mJy}$ for the flux expected from the core. Felli et al. (2004) estimate that the core diameter in the 
Table 2. MIR fluxes for the Spitzer-IRAC sources towards the mm core.

\begin{tabular}{lcccccc}
\hline \hline ID & \multicolumn{2}{c}{ Position } & $\begin{array}{c}F_{v}(3.6) \\
(\mathrm{mJy})\end{array}$ & $\begin{array}{c}F_{v}(4.5) \\
(\mathrm{mJy})\end{array}$ & $\begin{array}{c}F_{v}(5.8) \\
(\mathrm{mJy})\end{array}$ & $\begin{array}{c}F_{v}(8.0) \\
(\mathrm{mJy})\end{array}$ \\
\hline 1 & $05^{\mathrm{h}} 40^{\mathrm{m}} 54.2^{\mathrm{s}}$ & $35^{\circ} 41^{\prime} 59^{\prime \prime}$ & $8.6 \pm 0.8$ & $7.0 \pm 0.8$ & $<12$ & $<39$ \\
2 & $05^{\mathrm{h}} 40^{\mathrm{m}} 53.7^{\mathrm{s}}$ & $35^{\circ} 41^{\prime} 56^{\prime \prime}$ & $7.8 \pm 0.9$ & $4.7 \pm 1.2$ & $<11$ & $<19$ \\
3 & $05^{\mathrm{h}} 40^{\mathrm{m}} 53.5^{\mathrm{s}}$ & $35^{\circ} 41^{\prime} 53^{\prime \prime}$ & $17.2 \pm 0.3$ & $21.2 \pm 0.6$ & $29 \pm 1$ & $43 \pm 4$ \\
4 & $05^{\mathrm{h}} 40^{\mathrm{m}} 53.7^{\mathrm{s}}$ & $35^{\circ} 41^{\prime} 47^{\prime \prime}$ & $1.9 \pm 0.2$ & $2.9 \pm 0.4$ & $<3$ & $<5$ \\
5 (M 1) & $05^{\mathrm{h}} 40^{\mathrm{m}} 53.6^{\mathrm{s}}$ & $35^{\circ} 41^{\prime} 43^{\prime \prime}$ & $8.6 \pm 0.1$ & $15.0 \pm 0.1$ & $16 \pm 1$ & $13 \pm 1$ \\
6 & $05^{\mathrm{h}} 40^{\mathrm{m}} 54.0^{\mathrm{s}}$ & $35^{\circ} 41^{\prime} 41^{\prime \prime}$ & $2.1 \pm 0.1$ & $4.8 \pm 0.1$ & $9 \pm 1$ & $9 \pm 1$ \\
7 & $05^{\mathrm{h}} 40^{\mathrm{m}} 54.4^{\mathrm{s}}$ & $35^{\circ} 41^{\prime} 29^{\prime \prime}$ & $5.9 \pm 0.1$ & $7.5 \pm 0.1$ & $9 \pm 1$ & $10 \pm 1$ \\
8 & $05^{\mathrm{h}} 40^{\mathrm{m}} 53.8^{\mathrm{s}}$ & $35^{\circ} 41^{\prime} 27^{\prime \prime}$ & $33.6 \pm 0.1$ & $33.1 \pm 0.1$ & $36 \pm 1$ & $54 \pm 1$ \\
$9^{a}$ & $05^{\mathrm{h}} 40^{\mathrm{m}} 52.2^{\mathrm{s}}$ & $35^{\circ} 41^{\prime} 41^{\prime \prime}$ & $5.0 \pm 0.3$ & $4.7 \pm 0.2$ & $<5$ & $<24$ \\
10 & $05^{\mathrm{h}} 40^{\mathrm{m}} 52.7^{\mathrm{s}}$ & $35^{\circ} 41^{\prime} 45^{\prime \prime}$ & $8.5 \pm 0.2$ & $7.6 \pm 0.2$ & $9 \pm 1$ & $18 \pm 7$ \\
11 & $05^{\mathrm{h}} 40^{\mathrm{m}} 52.2^{\mathrm{s}}$ & $35^{\circ} 41^{\prime} 55^{\prime \prime}$ & $5.4 \pm 0.8$ & $2.9 \pm 0.7$ & $<17$ & $<95$ \\
12 & $05^{\mathrm{h}} 40^{\mathrm{m}} 51.7^{\mathrm{s}}$ & $35^{\circ} 41^{\prime} 57^{\prime \prime}$ & $3.5 \pm 0.8$ & $2.7 \pm 0.6$ & $<20$ & $<59$ \\
13 (S235AB-MIR) & $05^{\mathrm{h}} 40^{\mathrm{m}} 53.4^{\mathrm{s}}$ & $35^{\circ} 41^{\prime} 49^{\prime \prime}$ & $<0.6$ & $5.0 \pm 0.3$ & $14 \pm 1$ & $28 \pm 2$ \\
14 & $05^{\mathrm{h}} 40^{\mathrm{m}} 53.0^{\mathrm{s}}$ & $35^{\circ} 41^{\prime} 53^{\prime \prime}$ & $1.7 \pm 0.9$ & $3.7 \pm 1.0$ & $3 \pm 4$ & $<35$ \\
15 & $05^{\mathrm{h}} 40^{\mathrm{m}} 52.7^{\mathrm{s}}$ & $35^{\circ} 41^{\prime} 52^{\prime \prime}$ & $2.4 \pm 1.1$ & $1.2 \pm 0.9$ & $<9$ & $<22$ \\
\hline
\end{tabular}

${ }^{a} \mathrm{~N}-\mathrm{E}$ of a close by source only visible in the $5.8 \mu \mathrm{m}$ band.

continuum at the highest resolution $(1.2 \mathrm{~mm})$ is $\sim 3^{\prime \prime}$; hence, assuming that all the emission is uniformly distributed in a circle of 1 .' 5 in radius and using the synthesized beam size at $0.7 \mathrm{~cm}$ given in Table 1, we obtain an expected flux of $\sim 0.12 \mathrm{mJy} / \mathrm{beam}$, i.e. $<2 \sigma$ (see Table 1). Hence, dust emission at $0.7 \mathrm{~cm}$ could be present below the sensitivity limit of our observations.

Similarly, no extended emission from the jet was observed at any of our four frequencies. While this could be an effect of overresolution and low surface brightness of the jet at the shortest wavelengths, it definitely rules out the hypothesis of an ionized jet at 6 and $3.6 \mathrm{~cm}$. In fact, at $6 \mathrm{~cm}$, the flux density per beam area extrapolated from the $3.3 \mathrm{~mm}$ flux in the hypothesis of an ionized jet (i.e. using a spectral index $\alpha=0.6$ ) would be a factor of 3 higher than the upper limit quoted in Table 3.

At 1.3 and $0.7 \mathrm{~cm}$, where the resolution is higher, two nearly unresolved sources are present. They have been named VLA-1 (or S235AB-VLA-1) and VLA-2 (or S235AB-VLA-2) and are indicated in Fig. 6, where we show the VLA map at $1.3 \mathrm{~cm}$ (contours) overlaid on the Plateau de Bure map at $1.2 \mathrm{~mm}$ (grey scale) from Felli et al. (2004). We derived the integrated fluxes of the two sources within the $3 \sigma$ level on the maps obtained with natural weighting. At 3.6 and $6 \mathrm{~cm}$, the two sources fall within the sidelobes of $\mathrm{S} 235 \mathrm{~A}$. This results in a noise higher than that predicted from the total integration time (see Table 1). Using different weightings to partially filter out the extended emission does not yield any significant improvement in the measurements. The flux densities are listed in Table 3; the upper limits at $6 \mathrm{~cm}$ (for both) and at $3.6 \mathrm{~cm}$ (for VLA-2) refer to a point source.

VLA-1 lies $\sim 10^{\prime \prime}$ south of the mm core and is located within the jet, almost along its axis (see Figs. 1 and 6). It coincides with a small component of the fragmented jet observed at $1.2 \mathrm{~mm}$ and, most noticeably, with the $K$-band source $\mathrm{M} 1$.

VLA-2 lies within the boundary of the S235B nebulosity. VLA-2 represents the first radio detection of this peculiar region. Our flux densities of $\sim 0.5 \mathrm{mJy}$ (see Table 3 ) are not in conflict with the previously derived upper limits of $5 \mathrm{mJy}$ at $6 \mathrm{~cm}\left(10^{\prime \prime}\right.$ beam, Israel \& Felli 1978) and $0.3 \mathrm{mJy}$ at $3.6 \mathrm{~cm}$ (0.'1 beam, Tofani et al. 1995).

We have checked the probability that VLA-1 and VLA-2 are background sources. The expected number of extragalactic sources at $1.3 \mathrm{~cm}$ in the field of view of Fig. $6(\sim 1$ square arcmin) based on Eq. (A11) of Anglada et al. (1998) is $N \sim 0.05$, making this possibility very unlikely.

\section{3. $\mathrm{H}_{2} \mathrm{O}$ masers}

\subsubsection{VLA observations}

In the selected velocity range (roughly from -70 to $10 \mathrm{~km} \mathrm{~s}^{-1}$ ), three maser spots were detected above the $5 \sigma$ noise. We have determined the flux densities and positions of the maser spots by 2-dimensional Gaussian fits in each channel. All velocity components in the same spot are spatially unresolved. Coordinates of the three maser spots and flux density for each velocity peak are listed in Table 4; their relations to the other features present in the area are shown in Figs. 6 and 13.

It is important to note that the three maser spots cover different velocity ranges, as shown in Fig. 7, so that there is no velocity overlap among the three spatial components.

One of them (S235AB-H2O/3) coincides, within $\sim 0$.'5, with that found with the VLA at $\sim-60 \mathrm{~km} \mathrm{~s}^{-1}$ by Tofani et al. (1995) and emits in the same velocity range. The other two (S235AB$\mathrm{H} 2 \mathrm{O} / 1$ and S235AB-H2O/2) occur at radial velocities that had not been searched for in the previous VLA observation because at that time they were not detectable in single-dish observations, but they have since been revealed in the Medicina patrol.

The maser luminosity for each spot was obtained by integrating the line emission within the respective velocity ranges. The results are listed in Table 5, along with the corresponding velocity range. The $\mathrm{H}_{2} \mathrm{O}$ luminosity is typical of masers associated with far-Infrared (FIR) sources of $10^{3}-10^{4} L_{\odot}$ (see Palagi et al. 1993). This is consistent with the upper limit of the bolometric luminosity inferred for the mm core by Felli et al. (2004).

From Figs. 6 and 13, S235AB-H2O/2 clearly coincides with VLA-1 and M 1 and does not seem to be directly related to the $\mathrm{mm}$ core. The other two spots (S235AB-H2O/1 and $\mathrm{S} 235 \mathrm{AB}-\mathrm{H} 2 \mathrm{O} / 3$ ) are very close to the $\mathrm{mm}$ core and are perpendicularly aligned to the NE-SW outflow, $\sim 2$ " apart from each other. The possibility that they might be tracing a disk or torus perpendicular to the NE-SW outflow will be examined in Sect. 4. 
Table 3. Radio continuum fluxes. Upper limits are $3 \sigma$ noise levels.

\begin{tabular}{lcccccc}
\hline \hline Source & \multicolumn{2}{c}{ Position } & \multicolumn{4}{c}{$S_{v}(\mathrm{mJy})$} \\
& $\alpha(2000)$ & $\delta(2000)$ & $0.7 \mathrm{~cm}$ & $1.3 \mathrm{~cm}$ & $3.6 \mathrm{~cm}$ & $6 \mathrm{~cm}$ \\
\hline S235A & $05^{\mathrm{h}} 40^{\mathrm{m}} 52.70^{\mathrm{s}}$ & $35^{\circ} 42^{\prime} 21^{\prime \prime}$ & $114 \pm 3^{a}$ & $172 \pm 1$ & $248 \pm 2$ & $257 \pm 7$ \\
VLA-1 & $05^{\mathrm{h}} 40^{\mathrm{m}} 53.60^{\mathrm{s}}$ & $35^{\circ} 41^{\prime} 43^{\prime \prime}$ & $0.59 \pm 0.08$ & $0.44 \pm 0.03$ & $0.39 \pm 0.08$ & $<0.27$ \\
VLA-2 & $05^{\mathrm{h}} 40^{\mathrm{m}} 52.40^{\mathrm{s}}$ & $35^{\circ} 41^{\prime} 30^{\prime \prime}$ & $0.48 \pm 0.08$ & $0.47 \pm 0.03$ & $<0.24$ & $<0.27$ \\
\hline
\end{tabular}

${ }^{a}$ Corrected for primary beam attenuation.
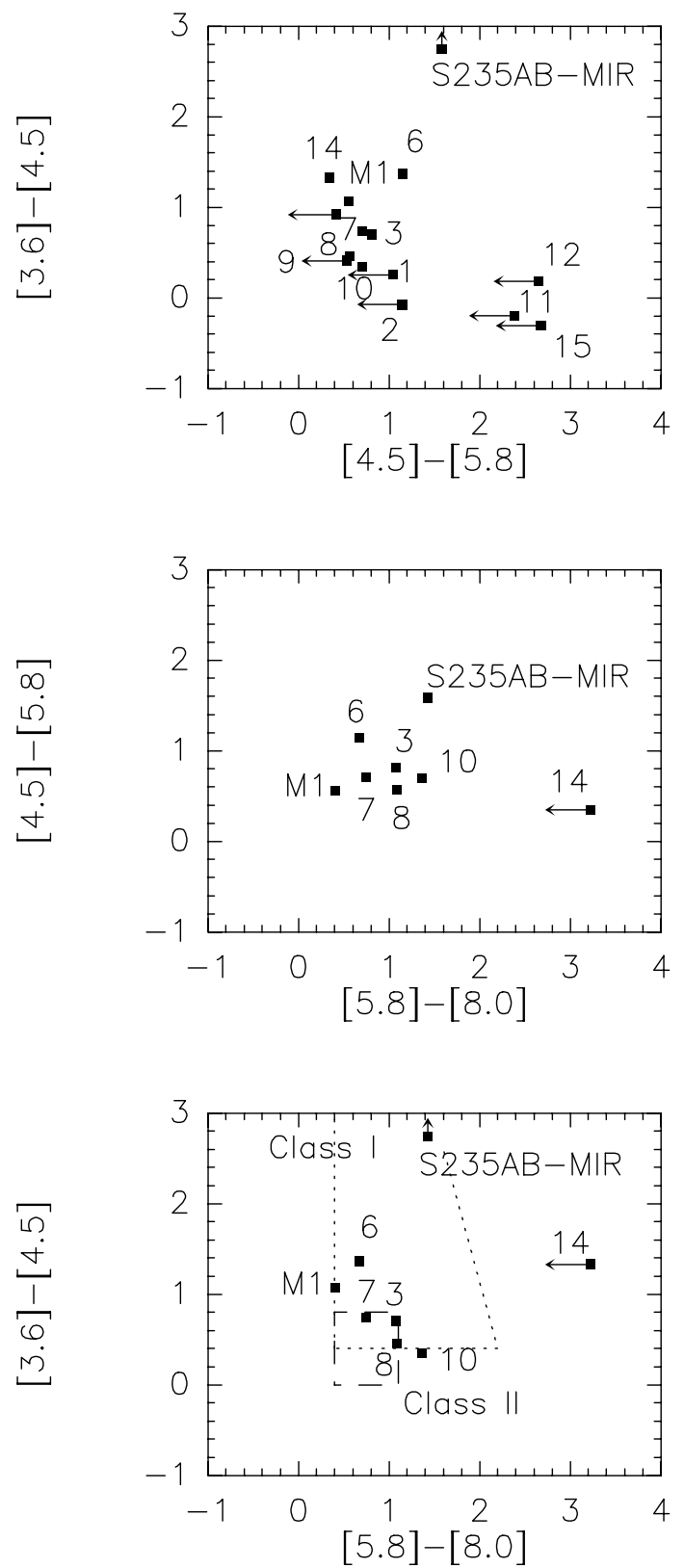

Fig. 4. Colour-colour plots of the most relevant sources in the area of the S235A-B cluster. The identifying numbers and symbols are the same as those used in Table 2 and Fig. 2. In the bottom box, the regions occupied by Class I and Class II sources are enclosed with dotted and dashed lines, respectively. When only an upper limit to the flux density could be estimated in one of the bands, the corresponding point in the plot is marked by an arrow.

\subsubsection{Medicina observations}

The different velocity ranges of the maser spots detected with the VLA make the low-resolution Medicina observations a

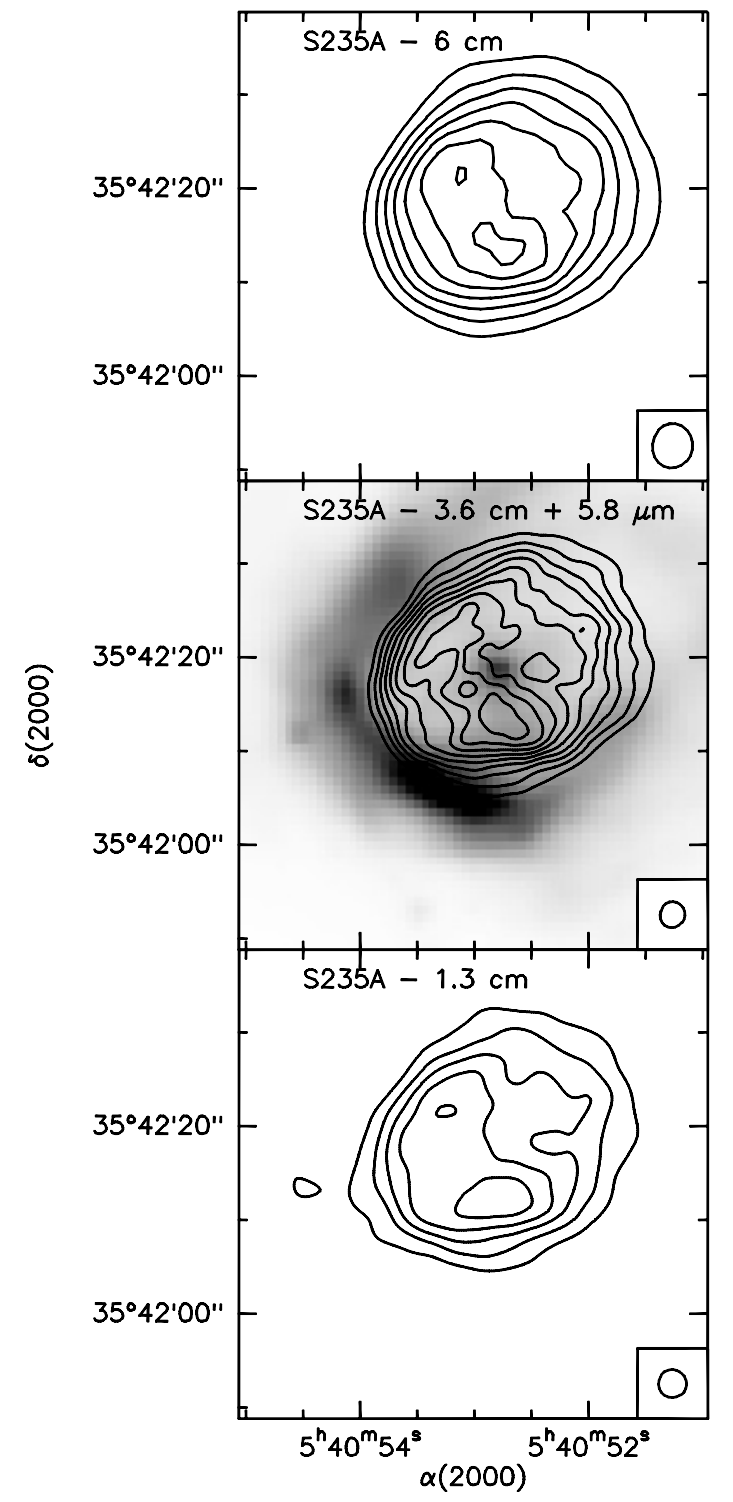

Fig. 5. Maps of the continuum radio emission from S235A at 6 , 3.6 , and $1.3 \mathrm{~cm}$ (contours). Levels are from 2 to $14 \mathrm{mJy} / \mathrm{beam}$ by $2 \mathrm{mJy} /$ beam for $6 \mathrm{~cm}$, from 1 to $5 \mathrm{mJy} /$ beam by $0.5 \mathrm{mJy} / \mathrm{beam}$ for $3.6 \mathrm{~cm}$, and 0.1 to $0.5 \mathrm{mJy} /$ beam by $0.1 \mathrm{mJy} /$ beam for $1.3 \mathrm{~cm}$. The $1.3 \mathrm{~cm}$ map has been smoothed to a lower resolution to increase the $\mathrm{S} / \mathrm{N}$ ratio. The $3.6 \mathrm{~cm}$ map is overlaid with the $5.8 \mu \mathrm{m}$ Spitzer-IRAC image (grey scale).

useful tool to follow the evolution of each spot, with no confusion arising from the fact that they are all within the Medicina beam. The upper envelope of the water maser emission using all the available single-dish observations is shown in Fig. 8. When compared with Fig. 7, it shows that the emissions from the three spatially separated VLA maser spots occur at very 


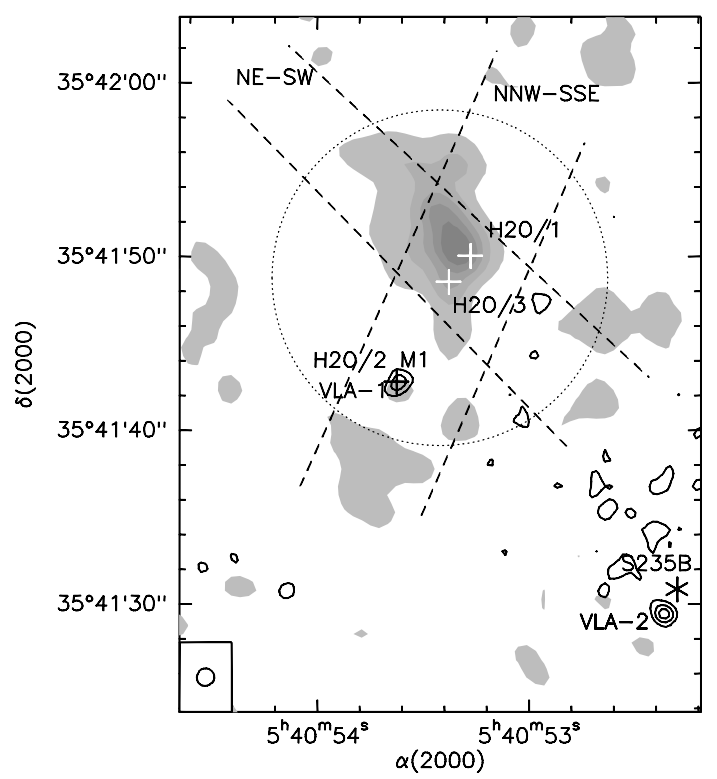

Fig. 6. Map of the continuum radio emission at $1.3 \mathrm{~cm}$ of the area around the water masers (contours) overlaid with the map of the continuum $1.2 \mathrm{~mm}$ emission (grey scale) from Felli et al. (2004). Levels are from $0.12(\sim 4 \sigma)$ to $0.48 \mathrm{mJy} / \mathrm{beam}$ in steps of $0.12 \mathrm{mJy} / \mathrm{beam}$. The locations of the three $\mathrm{H}_{2} \mathrm{O}$ maser spots detected in this work are indicated by "+". M 1 and the centre of S235B (asterisk) are also indicated. The dashed lines define the directions of the two outflows. The dotted circle is the primary beam at $1.2 \mathrm{~mm}$. The synthesized beam at $1.3 \mathrm{~cm}$ is drawn in the bottom left-hand corner.

Table 4. Water masers: fluxes and positions.

\begin{tabular}{ccccc}
\hline \hline Name & \multicolumn{2}{c}{ Position } & & \\
S235A-B & $\alpha(2000)$ & $\delta(2000)$ & $\begin{array}{c}V_{\text {LSR }} \\
\left(\mathrm{km} \mathrm{s}^{-1}\right)\end{array}$ & $\begin{array}{c}S_{v} \\
(\mathrm{Jy})\end{array}$ \\
\hline $\mathrm{H} 2 \mathrm{O} / 1$ & $05^{\mathrm{h}} 40^{\mathrm{m}} 53.27^{\mathrm{s}}$ & $35^{\circ} 41^{\prime} 50^{\prime} \cdot 0$ & 7 & 1.20 \\
& & & 3 & 0.50 \\
$\mathrm{H} 2 \mathrm{O} / 2$ & $05^{\mathrm{h}} 40^{\mathrm{m}} 53.63^{\mathrm{s}}$ & $35^{\circ} 41^{\prime} 43^{\prime \prime} \cdot 0$ & -18 & 0.16 \\
& & & -25 & 0.52 \\
& & & -29 & 1.66 \\
$\mathrm{H} 2 \mathrm{O} / 3$ & $05^{\mathrm{h}} 40^{\mathrm{m}} 53.38^{\mathrm{s}}$ & $35^{\circ} 41^{\prime} 48^{\prime \prime} 6$ & -58 & 0.37 \\
& & & -62 & 0.23 \\
& & & -64 & 0.22 \\
& & & -68 & 1.44 \\
\hline
\end{tabular}

different velocity ranges, being thus separated (although spatially unresolved) in the Medicina observations, as well.

In Fig. 9, we show the time-velocity-intensity plot from the Medicina patrol. An indicative value of the noise level in these observations throughout the entire period is of the order of 1-2 Jy. The starting date corresponds to March 31, 1987. The patrol is sparser at the beginning. After 1992, there are about 4 observations every year. Following the separation in velocity of the three maser spots, each velocity component (at $\sim-60, \sim-25$, and $\sim 0 \mathrm{~km} \mathrm{~s}^{-1}$ ) is labelled in Fig. 9 with the corresponding VLA name. The dates of the two VLA observations are indicated. The systemic velocity of the molecular cloud $\left(-17 \mathrm{~km} \mathrm{~s}^{-1}\right)$ is also indicated. Only S235AB-H2O/2 occurs at a velocity close to that of the thermal molecular lines; the other two components (S235AB-H2O/1 and S235AB-H2O/3) emit well outside the width of the thermal molecular lines.

The biggest flare occurred from the component S235AB$\mathrm{H} 2 \mathrm{O} / 3$ at the time of the first VLA observation. The source then

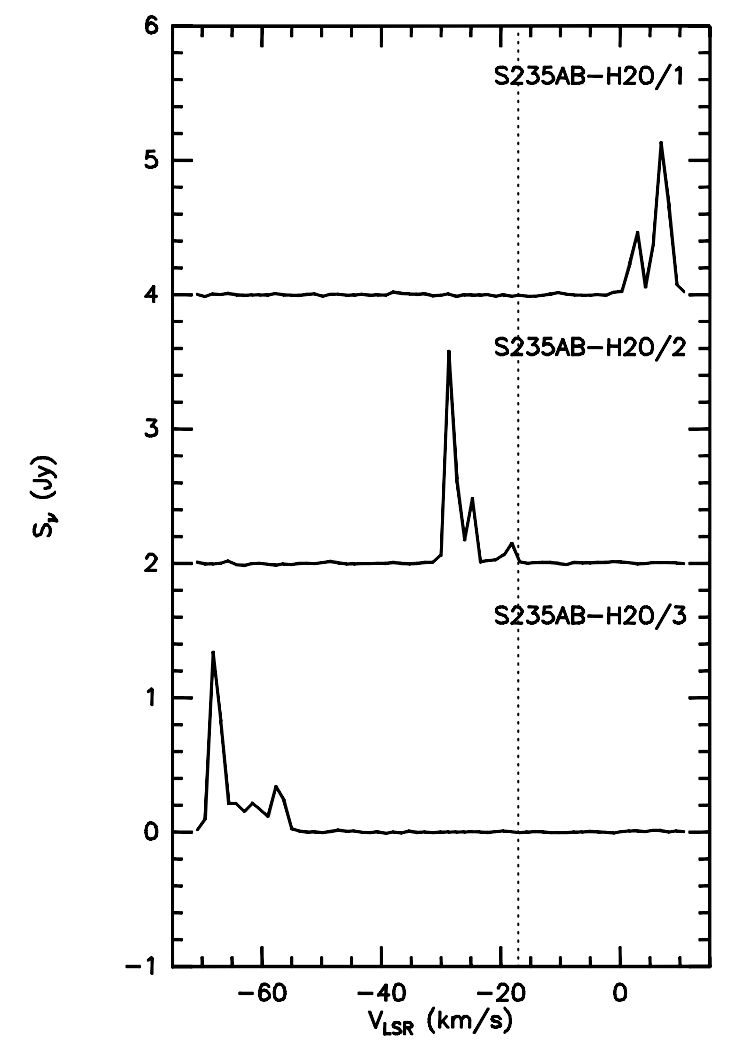

Fig. 7. $\mathrm{H}_{2} \mathrm{O}$ VLA spectra averaged on a circle $1^{\prime \prime}$ in radius and centred on the locations of each of the 3 maser spots. The name of the spot is indicated above the corresponding spectrum. The intensity scale is offset by 2 and $4 \mathrm{Jy}$ for clearness of the display. The vertical line defines the velocity of the molecular cloud.

disappeared below the noise and came up again just shortly before the second VLA observation.

Component S235AB-H2O/2 was undetectable for most of the time and appeared above the noise only after 2000.

Component S235AB-H2O/1, although always rather weak, is present most of the time. Its most noteworthy aspect is that the velocity changes up to $\pm 5 \mathrm{~km} \mathrm{~s}^{-1}$ around a mean value of $\sim 0 \mathrm{~km} \mathrm{~s}^{-1}$. To better illustrate this effect, the time-velocityintensity plot for the velocity range from -10 to $10 \mathrm{~km} \mathrm{~s}^{-1}$ is shown in Fig. 10.

To make sure that the change in velocity is a real effect and not an instrumental one and to provide an independent estimate of the accuracy on the velocity, we have examined another water maser (G32.74-0.08), that is also included in the Medicina patrol, and that was chosen because it is characterized by a single, narrow, and intense velocity component. For this maser, the velocity of the peak displays a maximum deviation from the mean value over the entire period of $<0.1 \mathrm{~km} \mathrm{~s}^{-1}$, a factor of 50 smaller than the velocity spread observed in S235AB-H2O/1.

\section{Discussion}

\section{1. $S 235 A$}

In Fig. 5, the overlay of the $3.6 \mathrm{~cm}$ map with the $5.8 \mu \mathrm{m}$ Spitzer image shows that the peak of the IR emission occurs in a shell outside the boundary of the radio emission. This proves that PAH and thermal dust emissions are mostly located beyond the ionization front in the Photodissociation Region. 
Table 5. Water masers: line luminosity.

\begin{tabular}{ccc}
\hline \hline Name & $\begin{array}{c}L_{\mathrm{H}_{2} \mathrm{O}}{ }^{a} \\
\left(10^{-7} L_{\odot}\right)\end{array}$ & $\begin{array}{c}\Delta V_{\mathrm{LSR}} \\
\left(\mathrm{km} \mathrm{s}^{-1}\right)\end{array}$ \\
\hline $\mathrm{S} 235 \mathrm{AB}-\mathrm{H} 2 \mathrm{O} / 1$ & 3.1 & 9 to -1 \\
$\mathrm{~S} 235 \mathrm{AB}-\mathrm{H} 2 \mathrm{O} / 2$ & 3.3 & -17 to -30 \\
$\mathrm{~S} 235 \mathrm{AB}-\mathrm{H} 2 \mathrm{O} / 3$ & 4.1 & -55 to -71 \\
\hline
\end{tabular}

${ }^{a}$ All masers are assumed to be at the distance of the S235A-B complex.

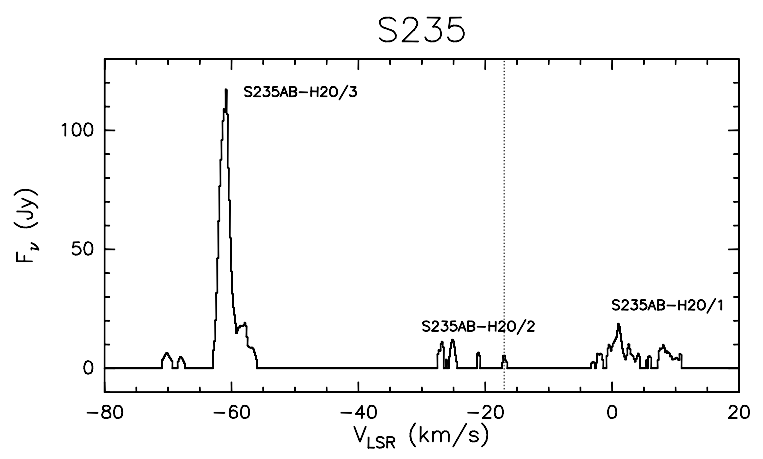

Fig. 8. Upper envelope of all water maser spectra observed with the Medicina radio telescope towards S235A-B, until July 2005. The three corresponding maser spots detected with the VLA observations are indicated. The vertical line defines the velocity of the molecular cloud.

In Fig. 11, we show the overlay of the $\mathrm{HCO}^{+}$integrated emission around $-17 \mathrm{~km} \mathrm{~s}^{-1}$ with the $5.8 \mu \mathrm{m}$ Spitzer-IRAC image. The overlay indicates that the lower contours of the molecular emission closely follow the outer boundary of S235A, suggesting that the HII region and the molecular cloud are interacting. It is also worth noting that the mm core is just outside the S235A boundary. This situation resembles that of some wellknown cometary-shaped UCHII regions such as G29.96-0.02 and G34.26+0.15 (Reid \& Ho 1985; Wood \& Churchwell 1989; Fey et al. 1995), which face a density peak of the molecular clump enshrouding them (Maxia et al. 2001; Gibb et al. 2004; Watt \& Mundy 1999). In a number of cases, it has been found that such a peak coincides with a hot molecular core, where massive star formation is going on (Cesaroni et al. 1998; Heaton et al. 1989; Garay \& Rodríguez 1990). In our case, the temperature of the molecular core is $\sim 30 \mathrm{~K}$ (see Felli et al. 2004), well below the typical temperature of hot molecular cores (see Kurtz et al. 2000), and the HII region S235A has an asymmetric rather than a cometary shape; nevertheless, the interaction between the ionized gas and the molecular cloud, as traced by the overall structure of the region, suggests that in this case also, one is observing an active burst of star formation where different evolutionary phases (from cores to evolved HII regions) co-exist. Whether the star formation episode in the molecular core has been triggered by the expansion of the nearby HII region S235A remains an open issue.

\subsection{VLA-1 and the jet}

Our radio continuum observations were unable to detect the elongated structure that had previously been found at $3.3 \mathrm{~mm}$, which also coincides with the blue lobe of the NNW-SSE $\mathrm{HCO}^{+}(1-0)$ outflow. Instead, a compact radio source, VLA-1, was found coincident with a small blob of emission in the central part of the $1.2 \mathrm{~mm}$ "jet", close ( 3 " east) to the weaker molecular peak at $-19 \mathrm{~km} \mathrm{~s}^{-1}$, called C 19 (Felli et al. 2004). More

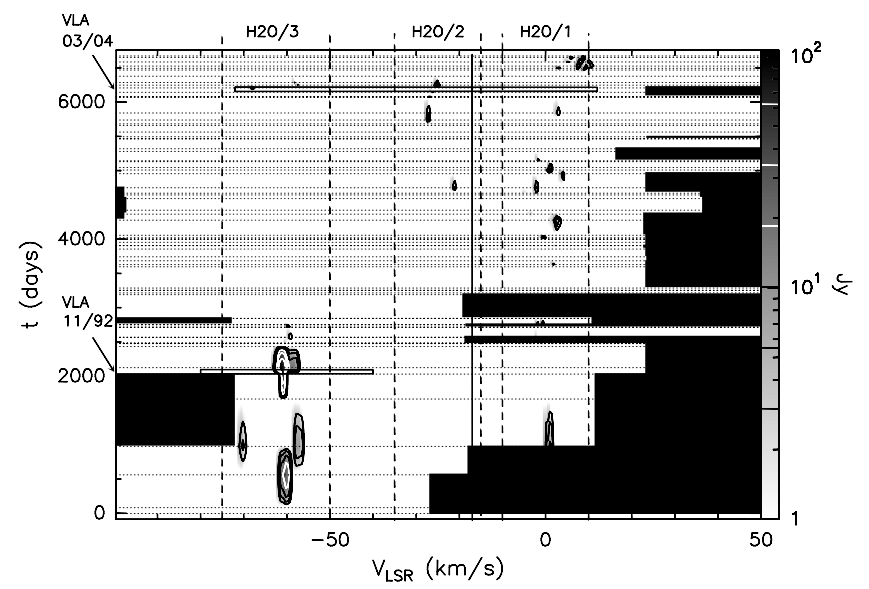

Fig. 9. Time-velocity-intensity plot of the water maser emission from S235A-B observed with the Medicina radio telescope. The starting date is March 31, 1987. The dates of the first VLA observation by Tofani et al. (1995) and of the present observations are indicated (month/year) with an arrow and their velocity ranges are enclosed within a long rectangle. The three maser spots are indicated by bracketing the velocity ranges with vertical dashed lines. The vertical solid line defines the velocity of the molecular cloud. The black areas are time-velocity regions with no observations.

importantly, VLA-1 coincides with the newly found water maser S235AB-H2O/2 and with $\mathrm{M} 1$, as shown in Fig. 12, where the $1.3 \mathrm{~cm}$ VLA map is overlaid on the Spitzer-IRAC $3.6 \mu \mathrm{m}$ image. M 1, which had been previously assumed to be associated with the $-60 \mathrm{~km} \mathrm{~s}^{-1}$ water maser (Felli et al. 1997), is instead associated with a radio continuum source and a new, separate water maser at $\sim-30 \mathrm{~km} \mathrm{~s}^{-1}$.

Although the errors on the flux densities are large, the values given in Table 3 for VLA- 1 are consistent with a spectral index $\alpha \approx 0.2$. This value is typical of partially optically thick free-free emission.

Since the present morphology no longer supports an ionized jet interpretation, we have to consider the alternative possibility that VLA-1 is an independent UCHII region, a hypothesis that should also be considered, in light of the precise association of the water maser with M 1. A lower limit to the total number $N_{\text {Ly }}$ of ionizing photons can be obtained from the $1.3 \mathrm{~cm}$ radio flux (the one with the highest signal-to-noise ratio) by assuming that the HII regions are optically thin (Mezger 1978). We obtain $N_{\mathrm{Ly}} \sim 10^{44} \mathrm{~s}^{-1}$, typical of B2-B3 ZAMS stars (Panagia 1973).

What may have caused the apparent disagreement between the $\mathrm{cm}$ radio observations which postulate the presence of a UCHII region and the mm observation that had suggested a jet? Extrapolating the radio flux of VLA-1 according to $v^{\alpha}(\alpha \approx 0.2)$, we derive $\sim 0.8 \mathrm{mJy}$ at $1.2 \mathrm{~mm}$ and $\sim 0.6 \mathrm{mJy}$ at $3.3 \mathrm{~mm}$. The $1.2 \mathrm{~mm}$ flux is below $1 \sigma(\sim 1 \mathrm{mJy})$ in the Plateau de Bure map, so it could not have been detected as a point source. The $1.2 \mathrm{~mm}$ flux of the "jet", $13 \mathrm{mJy}$, was integrated over a much larger area defined by the $3.3 \mathrm{~mm}$ map. At $3.3 \mathrm{~mm}$, the extrapolated flux of VLA- 1 is at a $2 \sigma$ level, again barely detectable as a point source, and in any case difficult to see since it would lie within the elongated $3.3 \mathrm{~mm}$ structure (about $10^{\prime \prime}$ in the elongated direction and unresolved in the perpendicular direction), with $7 \mathrm{mJy}$ of integrated flux.

These contradictory aspects can be reconciled if the emission from the elongated $3.3 \mathrm{~mm}$ structure comes from dust, perhaps that associated with the NNW-SSE blue outflow lobe or with the 


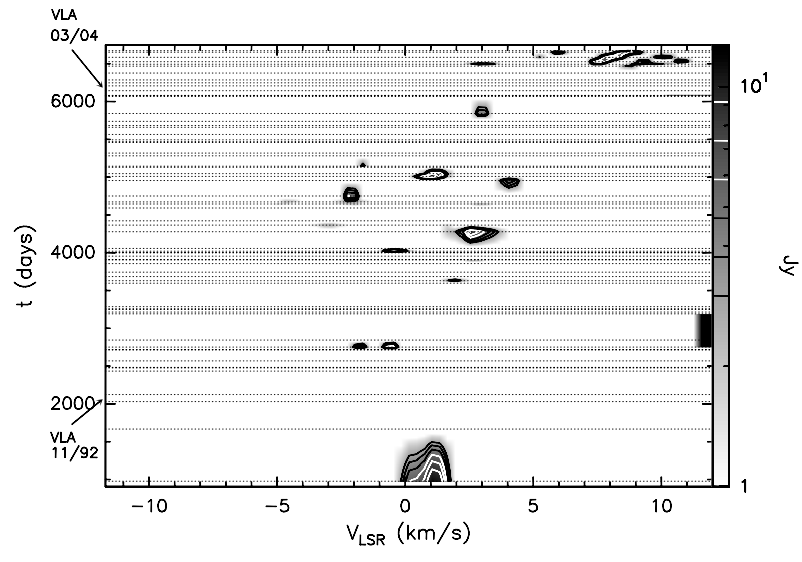

Fig. 10. Time-velocity-intensity plot of the water maser emission from S235A-B observed with the Medicina radio telescope over the velocity range from -10 to $+10 \mathrm{~km} \mathrm{~s}^{-1}$, which corresponds to the maser spot $\mathrm{S} 235 \mathrm{AB}-\mathrm{H} 2 \mathrm{O} / 1$.

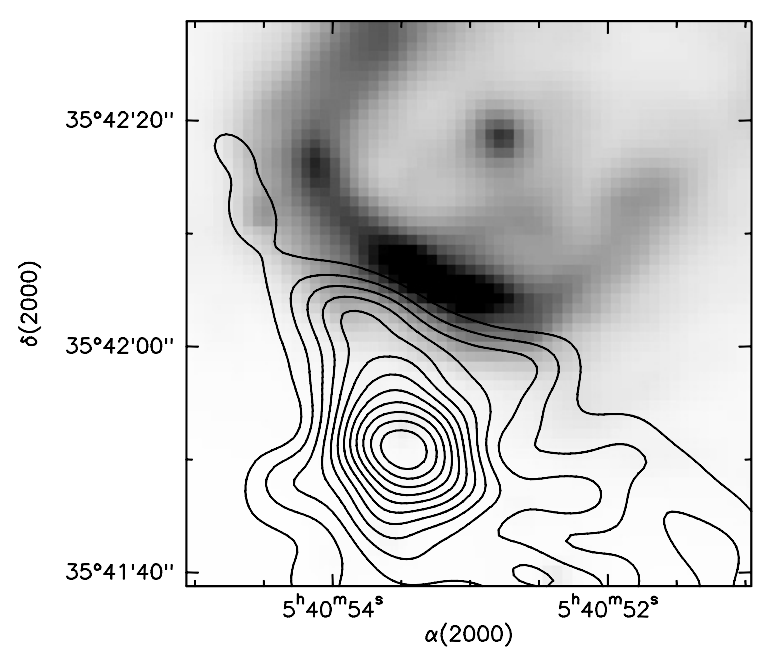

Fig. 11. The $\mathrm{HCO}^{+}$cloud at $-17 \mathrm{~km} \mathrm{~s}^{-1}$ (contours) overlaid on the $5.8 \mu \mathrm{m}$ Spitzer-IRAC image (grey scale). Note that the lower contours of the molecular emission closely follow the boundary of the IR emission, suggesting that the S235A HII region is interacting with the molecular cloud.

C 19 molecular peak. The spectral index $\alpha=0.6$ between 3.3 and $1.2 \mathrm{~mm}$ could be the result of the simultaneous presence of a dusty jet with a steep spectral index and a weak UCHII region showing up only at lower frequencies, where dust emission is negligible.

Is VLA-1/M 1 the driving source of the NNW-SSE outflow? Due to the overlap with the NE-SW outflow, the centre of the NNW-SSE outflow is ill-defined and any argument based on the position of VLA-1 with respect to the centre of the NNW-SSE outflow is inconclusive. However, we can exclude any association of VLA-1 with the NE-SW outflow, given its offset with respect to its axis.

Finally, we note that one of the methanol masers $\left(\mathrm{CH}_{3} \mathrm{OH} / 4\right)$ lies close ( $2^{\prime \prime}$ southwest) to VLA-1 and the water maser S235AB-H2O/2 (see Fig. 13) and has velocities in a similar range (from -16 to $-21 \mathrm{~km} \mathrm{~s}^{-1}$, see Kurtz et al. 2004), suggesting a common origin. The velocities of the water and methanol masers are very close to that of the molecular core, indicating that in this case, the component of the motions along the line of sight of the masers with respect to the molecular core is negligible.

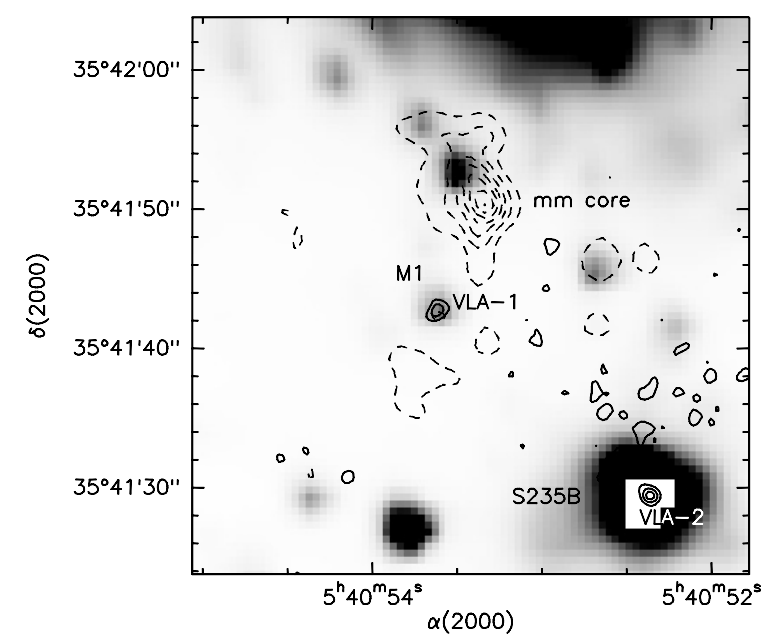

Fig. 12. Overlay of the $1.3 \mathrm{~cm}$ VLA map (full contours) with the Spitzer-IRAC $3.6 \mu \mathrm{m}$ image (grey scale) and the $1.2 \mathrm{~mm}$ continuum (dashed contours).

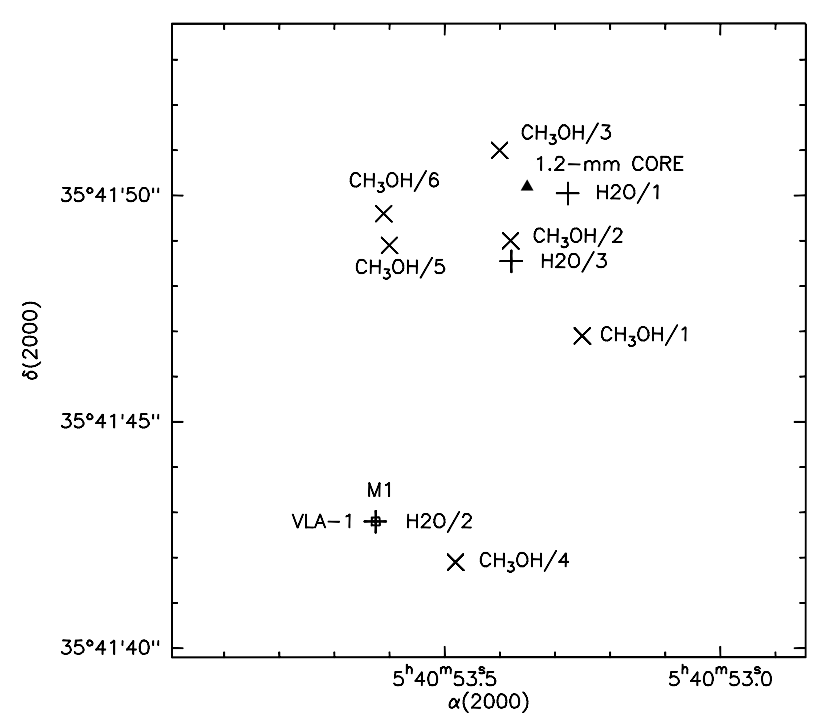

Fig. 13. Position of the three water masers $(\mathrm{H} 20 / 1-3+)$ and of the six methanol masers $(\mathrm{CH} 3 \mathrm{OH} / 1-6 \times)$ detected by Kurtz et al. (2004). The $1.2 \mathrm{~mm}$ core (triangle), VLA-1 (square), and M 1 are also indicated. Note that the symbol used to mark the location of the $1.2 \mathrm{~mm}$ core is smaller than the errorbars.

\subsection{VLA-2 and S235B}

The overlay of Fig. 12 shows that VLA-2 lies at the centre of the S235B bright diffuse nebula (saturated in all IRAC bands). The Spitzer-IRAC images, in particular those at longer wavelengths, indicate that this nebula is composed of two very close components (see Fig. 2). VLA-2 lies at the centre of the southern and more extended one. Overall, the size of the mid-IR nebula is about $10^{\prime \prime}$, similar to that observed in $\mathrm{H} \alpha$, so that the optical-IR morphology is more reminiscent of S235A (resolved HII region) than that of VLA-1 (IR and radio unresolved).

Besides being detected in $\mathrm{H} \alpha$, strong $\mathrm{Br} \gamma$ emission from an unresolved source (i.e. $<3^{\prime \prime}$ ) coincident with the $K$-band point source in S235B had been reported by Krassner et al. (1982) and Felli et al. (1997), with an integrated line flux of $F(\mathrm{Br} \gamma)=$ $(2.0 \pm 0.4) \times 10^{-12} \mathrm{erg} \mathrm{s}^{-1} \mathrm{~cm}^{-2}$. The expected radio flux density from an optically thin HII region at $6 \mathrm{~cm}$ would have been greater than $200 \mathrm{mJy}$, where the lower limit accounts for the fact 
that the Br $\gamma$ flux was not corrected for extinction. This is at odds with lower limits to the radio flux found in all previous radio continuum observations, as well as with the present detection. In the past, this forced abandon of the hypothesis of a classical HII region (unless extremely optically thick) suggested that the $\mathrm{Br} \gamma$ emission originates from an ionized expanding envelope around an early type star (Felli et al. 1997), in which case the ratio of radio-to-IR line emission would be reduced by about two orders of magnitude (Simon et al. 1983).

Our measured fluxes represent the first detection of the radio continuum from an underlying unresolved source. The radio flux density at $3.6 \mathrm{~cm}$ expected from a fully ionized envelope using $F(\operatorname{Br} \alpha) / F(\operatorname{Br} \gamma) \sim 0.8$ and Eq. (20) of Simon et al. (1983) is $0.46 \mathrm{mJy}$. While this agreement with the observed value might be fortuitous in view of the many unknown parameters involved (velocity of the wind, correction for extinction, etc.) it clearly points out that an ionized envelope around a lower luminosity, more-evolved star remains the best interpretation. However, it must be noted that the observed spectral index is smaller than the expected 0.6 value.

Our flux densities are fully consistent with the previous upper limits at radio wavelengths and with the unresolved nature of S235B. The implied mass loss using Eq. (14) of Felli \& Panagia (1981) is $4 \times 10^{-6} M_{\odot} \mathrm{yr}^{-1}$, quite large and indicative of a luminous star. Future radio recombination line observations with sufficiently high sensitivity may permit measurement of the line width to determine the velocity of the ionized wind. There are no masers, molecular peaks, outflows, or mm peaks associated with S235B, all of which suggest that S235B-VLA-2 is more evolved than the mm core and VLA-1.

The diffuse $\mathrm{H} \alpha$ and IR emission from S235B may be attributed to reflected light from ionized stellar envelopes.

Finally, what is the luminosity and mass of the star embedded in S235B? We cannot use the Spitzer-IRAC observations because they are saturated. Instead, we used the $J, H$, and $K$ magnitudes from Felli et al. (1997) and the MSX fluxes (see Egan et al. 1999). The integral of these values gives $410 L_{\odot}$, which must be considered to be a lower limit because the FIR part of the spectrum is not taken into account in our calculation.

\subsection{The $m m$ core and the S235AB-H2O/1 and S235AB-H2O/3 masers}

As was already pointed out, an important result of the $\mathrm{cm}$ radio continuum observations is the lack of emission from the $\mathrm{mm}$ core. In Felli et al. (2004), the luminosity of the embedded YSO had been estimated to be $10^{3} L_{\odot}$. If this comes from a ZAMS star of spectral type B3 or earlier, to make the radio free-free emission undetectable, the radius of the associated HII region must be less than $\sim 6.5 \mathrm{AU}$ due to confinement by a density larger than $7 \times 10^{6} \mathrm{~cm}^{-3}$. In any case, the detection of pure thermal dust emission from the core indicates a very early evolutionary phase of the embedded YSO.

The new result provided by the Spitzer-IRAC observations is the detection of S235AB-MIR very close to the mm peak. The positions of the mm core and S235AB-MIR differ by $\sim 1^{\prime \prime} .5$, which is slightly greater than the error on the relative positions, so that at this stage it cannot be firmly established if S235ABMIR represents the YSO embedded in the $\mathrm{mm}$ core. Its position in the colour-colour plots of Fig. 4 definitely puts this source in the Class I category.

It is possible to check whether the S235AB-MIR fluxes between $3.6-8 \mu \mathrm{m}$ are consistent with the spectral energy distribution (SED) of a heavily embedded (proto-)star of $10^{3} L_{\odot}$.

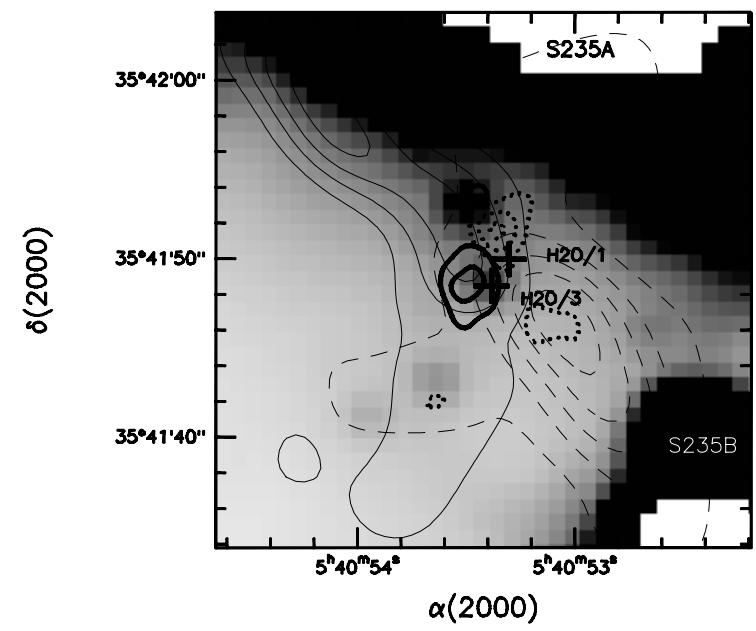

Fig. 14. $\mathrm{HCO}^{+}(1-0)$ emission from the NE-SW outflow (thin contours) overlaid with the blue- and red-shifted emission of $\mathrm{C}^{34} \mathrm{~S}(5-4)$ (thick contours) from Felli et al. (2004). The "+" symbols mark the location of the water maser spots $\mathrm{H} 2 \mathrm{O} / 1\left(\sim 7 \mathrm{~km} \mathrm{~s}^{-1}\right)$ and $\mathrm{H} 2 \mathrm{O} / 3\left(\sim-60 \mathrm{~km} \mathrm{~s}^{-1}\right)$. The background is the $8.0 \mu \mathrm{m}$ Spitzer-IRAC image (grey scale) and shows the position of S235AB-MIR, coincident with the southern water maser $(\mathrm{H} 2 \mathrm{O} / 3)$.

Felli et al. (2004) show that the non-detection of such a source in the $K$ band towards the mm core implies $A_{\mathrm{V}} \gtrsim 37 \mathrm{mag}$, in agreement with the derived $\mathrm{H}_{2}$ column density. Using the extinction law found by Indebetouw et al. (2005), we derived upper limits for the intrinsic fluxes (or upper limits) of S235ABMIR in the four IRAC bands. Of course, S235AB-MIR being a Class I source, most of the emission at these wavelengths arises from circumstellar matter rather than from the (proto-)star photosphere. For this reason, we compared our results to the SEDs models for intermediate-mass Class I sources (star plus disk and envelope, Whitney et al. 2003; Whitney et al. 2004). We found that, within the limits of the many parameters of their models, the MIR SED of S235AB-MIR is consistent with a central star later than B3.

The two northernmost water masers, S235AB-H2O/1 and $\mathrm{S} 235 \mathrm{AB}-\mathrm{H} 2 \mathrm{O} / 3$, are located close to the $\mathrm{mm}$ core and emit over velocity ranges quite different from those of the other water and methanol masers, as well as those of the molecular cloud $\left(\sim-17 \mathrm{~km} \mathrm{~s}^{-1}\right)$. Figure 14 (adapted from Fig. 21 of Felli et al. 2004) shows the NE-SW $\mathrm{HCO}^{+}$outflow with the contours of a perpendicular bipolar structure traced by the wings of $C^{34} S(5-4)$. This was interpreted by Felli et al. (2004) as the signature of a rotating disk around the YSO driving the outflow. Strikingly enough, the two water masers are aligned in the same direction and very close to the $\mathrm{C}^{34} \mathrm{~S}(5-4)$ structures: the blueshifted maser $\left(\mathrm{S} 235 \mathrm{AB}-\mathrm{H} 2 \mathrm{O} / 3\right.$ at $\sim-60 \mathrm{~km} \mathrm{~s}^{-1}$ ) lying towards the blue $\mathrm{C}^{34} \mathrm{~S}(5-4)$ lobe (integrated from -21 to $-19 \mathrm{~km} \mathrm{~s}^{-1}$ ) and the red-shifted maser (S235AB-H2O/1 at $\sim 7 \mathrm{~km} \mathrm{~s}^{-1}$ ) lying towards the red $\mathrm{C}^{34} \mathrm{~S}(5-4)$ lobe (from -16 to $-14 \mathrm{~km} \mathrm{~s}^{-1}$ ). However, in both cases the velocity of the water masers are more red or blue shifted than the corresponding $\mathrm{C}^{34} \mathrm{~S}(5-4)$ lobes.

A simple calculation based on the maser velocities shows that they cannot simply belong to a disk in Keplerian rotation around the YSO. From the difference between the most extreme maser velocities ( 7 and $-68 \mathrm{~km} \mathrm{~s}^{-1}$ ), we can infer a lower limit to the rotation velocity of $37 \mathrm{~km} \mathrm{~s}^{-1}$. If one assumes the halfdistance between the two maser spots $\left(\sim 1^{\prime \prime}\right.$ or $\left.1800 \mathrm{AU}\right)$ to be the orbital radius, then the mass needed to maintain such a rotating disk should be $>2500 M_{\odot}$, much larger than the mass of the molecular core. 
This fact proves that the water masers cannot trace rotation about an embedded YSO. One possibility is that they are instead the signature of the interaction with the $C^{34} S$ disk of high velocity outflowing material from the YSO. The higher (red and blue) velocities of the water maser with respect to $C^{34} S$ and their closer position to the $\mathrm{mm}$ core may suggest that the acceleration of the outflowing material occurs in the immediate surroundings of the YSO.

Another possibility is that the $\mathrm{C}^{34} \mathrm{~S}$ emission is not tracing a disk, but rather an outflow, whose high-velocity component would be seen in the $\mathrm{H}_{2} \mathrm{O}$ maser lines. This scenario is more consistent with the common belief that water masers are strictly associated with jets powering molecular outflows (Felli et al. 1992), as observed, e.g., in the massive protostar IRAS 20126+4104 (Moscadelli et al. 2000, 2005). If this hypothesis is correct, it remains to be established whether the bipolar outflow seen in the $\mathrm{C}^{34} \mathrm{~S}$ and $\mathrm{H}_{2} \mathrm{O}$ lines would be the same as the NNW-SSE outflow or a distinct one oriented approximately in the same direction, but originating from a different YSO.

Of the two hypotheses presented above, we believe that the outflow origin for the $\mathrm{C}^{34} \mathrm{~S}$ and $\mathrm{H}_{2} \mathrm{O}$ emission is the most likely, given the tight association between outflows and water masers. However, at present it is impossible to rule out the possibility that the $\mathrm{C}^{34} \mathrm{~S}$ emission is coming from a disk, as in the case of the Keplerian disk in IRAS 20126+4104 (Cesaroni et al. 2005). Class I methanol masers are believed to be excited in jets, so that a priori the $7 \mathrm{~mm} \mathrm{CH} \mathrm{CH}_{3} \mathrm{OH}$ masers imaged by Kurtz et al. (2004) in S235 could be used to establish the direction of the outflow and hence choose between the two hypotheses. As shown in Fig. 13, five of the maser spots cluster around the mm core suggesting that for them, too, the main source of energy is the YSO within the mm core. In particular, $\mathrm{CH}_{3} \mathrm{OH} / 2$ is very close to $\mathrm{S} 235 \mathrm{AB}-\mathrm{H} 2 \mathrm{O} / 3$. However their velocities lie within a narrow range (from -15.9 to $-21.0 \mathrm{~km} \mathrm{~s}^{-1}$ ) and the spots do not show a clear bipolarity, either in velocity or in their distribution. It is therefore difficult to associate the methanol maser emission to any precise geometry. The only conclusion is that they are unlikely to have the same dynamical origin as the two water masers.

\subsection{Water maser variability}

Some indication of what occurs in the immediate surroundings of the YSO embedded within the mm core may come from the variability of the two associated water masers.

The emission at $\sim-60 \mathrm{~km} \mathrm{~s}^{-1}(\mathrm{~S} 235 \mathrm{AB}-\mathrm{H} 2 \mathrm{O} / 3)$ reached its maximum (up to $\sim 110 \mathrm{Jy}$ ) in 1992-1993, lasting at most 2 years, then disappearing for most of the Medicina patrol, and finally reappearing just before the VLA observations.

Most noteworthy is the emission from S235AB-H2O/1 (between -10 and $10 \mathrm{~km} \mathrm{~s}^{-1}$ ), not only because of its long lasting presence and high variability, but also because of its velocity shifts, $\pm 5 \mathrm{~km} \mathrm{~s}^{-1}$. Figure 15 shows the LSR velocities of S235AB-H2O/1 (obtained by Gaussian fitting) in the period 1989-2005.

While it is possible that the velocity variations are simply due to the random flaring of spots at different velocities, we shall investigate more physically appealing interpretations.

To check whether the effect is due to a rotational modulation, we tried to fit all the observed velocities (the source is above the noise for about $60 \%$ of the time) with a sine function, as would be expected for a maser spot on a rotating disk viewed edge-on. No significant evidence was found.

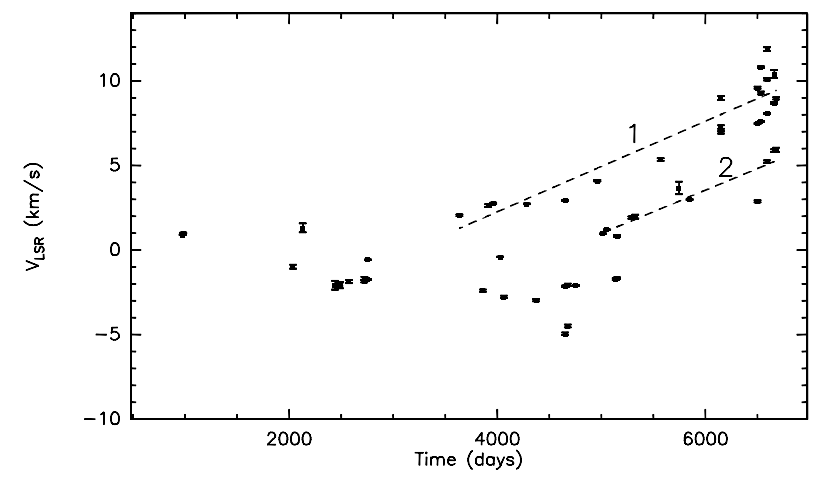

Fig. 15. Velocities of the peaks of S235AB-H2O/1 from Gaussian fits to the data from the Medicina radio telescope. We have tentatively outlined, with dashed lines, two components (labelled as 1 and 2) whose velocities might be drifting during the period of our observations.

The velocities in Fig. 15 are all redshifted with respect to the systemic velocity, but they are not all at random and seem to fan out at late periods. We identify two groups of points (labelled as 1 and 2 in Fig. 15), which exhibit linear drifts of velocity away from the systemic value. A linear fit (dashed lines in Fig. 15) provides velocity drifts of 0.93 and $0.98 \mathrm{~km} \mathrm{~s}^{-1} \mathrm{yr}^{-1}$, respectively. Velocity drifts of this amount have been observed in other water masers that are stronger and less variable in intensity (Brand et al. 2003). It is tempting to explain the velocity drifts with shocked material that is accelerated from a mean velocity $\sim 0 \mathrm{~km} \mathrm{~s}^{-1}$ by mass outflow from a central YSO. The lifetime of the accelerated spots is $\sim 1000-2000$ days ( $~ 3-6$ yrs) and could be related to the duration of ejection events from the YSO.

The maser outflows from S235AB-H2O/1 clearly deserve further, proper motion studies with VLBI techniques.

\section{Summary and conclusions}

We have presented new, more sensitive high-resolution VLA cm radio observations of the $\mathrm{S} 235 \mathrm{~A}-\mathrm{B}$ region, as well as the results of the Medicina water maser patrol (started in 1987), and archive Spitzer-IRAC observations. Several new aspects of this star-forming region emerge; they are illustrated in Fig. 16 and summarised in the following:

1. The radio-IR morphology of the S235A HII region confirms that it is a classical HII region, optically thin in the $\mathrm{cm}$ range. It appears to interact with the molecular cloud and may have induced the formation of a second generation YSO in the $\mathrm{mm}$ core.

2. No cm continuum emission is detected from the molecular core discovered by Felli et al. (2004). The lack of ionized hydrogen emission suggests a very early evolutionary phase for the intermediate-luminosity embedded YSO, much before the appearance of a UCHII region. We have found a new source, S235AB-MIR, detectable only at $4.5,5.8$, and $8.0 \mu \mathrm{m}$ and close to the $\mathrm{mm}$ core, in the archival Spitzer images. Given its position in the colour-colour plot, this could be the mid IR counterpart of the embedded YSO.

3. We have observed no extended $\mathrm{cm}$ continuum emission from the elongated jet-like structure detected at $3.3 \mathrm{~mm}$, suggesting that the putative $3.3 \mathrm{~mm}$ "jet" is due to dust and not to an ionized jet.

4. We found two compact radio-sources: VLA-1 and VLA-2. Their spectral index is suggestive of partially thick free-free emission. 


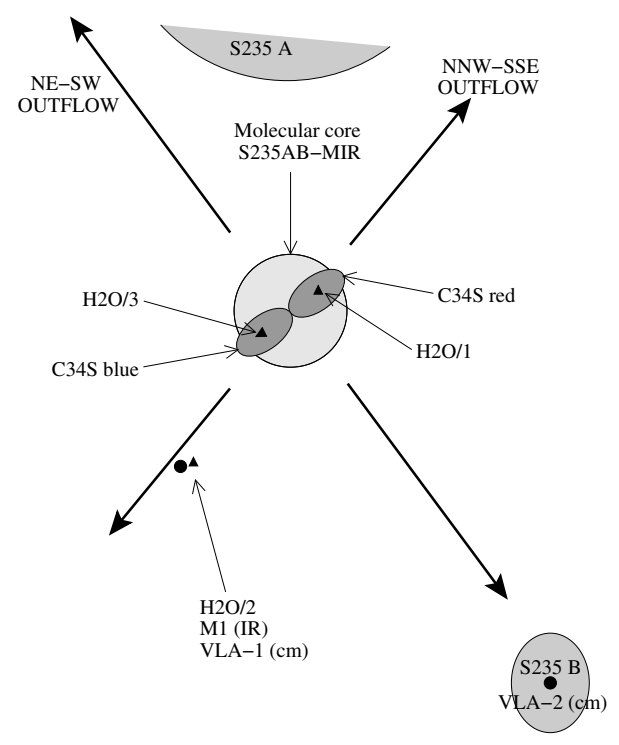

Fig. 16. Sketch (not to scale) of the star-forming region S235A-B in light of the new data presented in this paper. New and already-known sources are labelled, and their relationships are discussed in the text.

5. VLA-1 is located at the centre of the elongated $3.3 \mathrm{~mm}$ structure and coincides with the near-IR source M 1. It is close to the secondary molecular peak at $-19 \mathrm{~km} \mathrm{~s}^{-1}$. We estimate that VLA-1 could be a UCHII region associated with a B2-B3 star.

6. We have discovered a water maser $(\mathrm{S} 235 \mathrm{AB}-\mathrm{H} 2 \mathrm{O} / 2)$ at the same location of VLA-1. A methanol maser $\left(\mathrm{CH}_{3} \mathrm{OH} / 4\right)$ lies close by at a similar velocity.

7. VLA-2 is at the centre of S235B and represents the first radio continuum detection from this source. Comparison with the near-IR hydrogen lines confirms that both emissions come from an ionized envelope.

8. Two water masers (S235AB-H2O/1 and S235AB-H2O/3), with very different velocities $\left(\sim 7\right.$ and $\sim-60 \mathrm{~km} \mathrm{~s}^{-1}$, respectively), are located close to the $\mathrm{mm}$ core and are aligned parallel to a structure found in $\mathrm{C}^{34} \mathrm{~S}$ and perpendicular to the NE-SW outflow.

9. Our single-dish observations with the Medicina radio telescope do not spatially resolve the three water masers detected with the VLA, although do provide variability information since they do not overlap in velocity.

10. A high degree of variability in the water maser emission was found in all cases. We found changes of the LSR velocity with respect to the systemic velocity, up to $\sim 5 \mathrm{~km} \mathrm{~s}^{-1}$ for $\mathrm{S} 235 \mathrm{AB}-\mathrm{H} 2 \mathrm{O} / 1$. A possible interpretation would be velocity drifts due to shocked gas accelerated by the flaring activity of the YSO.

11. The duration of the acceleration, of an order of 3-6 yrs, is similar to the lifetime of the emission.

This paper, together with the preceding ones resulting from our long-term study, reveals the simultaneous presence in the S235A-B complex of widely different evolutionary phases, from the well-developed HII region S235A, to the peculiar object S235B-VLA-2, to the UCHII region VLA-1, to the $\mathrm{mm}$ core which harbors a YSO in a very early stage. The comparison of high resolution multiwavelength observations, combined with long-term monitoring of time variable phenomena, provides unique information on the nature of young (proto)stars, shedding new light on their interaction with the placental environment.
Acknowledgements. The Medicina observations are part of a long lasting project carried out by the Arcetri-INAF and IRA-INAF water maser group (see e.g. Brand et al. 2003, and references therein). This work is based in part on observations made with the Spitzer Space Telescope, which is operated by the Jet Propulsion Laboratory, California Institute of Technology under a contract with NASA. This research made use of data products from the Midcourse Space Experiment (MSX). Processing of the data was funded by the Ballistic Missile Defense Organization with additional support from the NASA Office of Space Science. We acknowledge G. Comoretto and F. Palagi for their help in the study of the variability of the water masers.

\section{References}

Allen, L. E., Calvet, N., D’Alessio, P., et al. 2004, ApJS, 154, 363 Alvarez, C., Hoare, M., Glindemann, A., \& Richichi, A. 2004, A\&A, 427, 505 Anglada, G., Villuendas, E., Estalella, R., et al. 1998, AJ, 116, 2953 Beltrán, M. T., Estalella, R., Anglada, G., Rodríguez, L. F., \& Torrelles, J. M. 2001, AJ, 121, 1556

Brand, J., Cesaroni, R., Comoretto, G., et al. 2003, A\&A, 407, 573

Cesaroni, R., Hofner, P., Walmsley, C. M., \& Churchwell, E. 1998, A\&A, 331, 709

Cesaroni, R., Felli, M., \& Walmsley, C. M. 1999, A\&AS, 136, 333

Cesaroni, R., Neri, R., Olmi, L., et al. 2005, A\&A, 434, 1039

Churchwell, E. 2002, ARA\&A, 40, 27

Comoretto, G., Palagi, F., Cesaroni, R., et al. 1990, A\&AS, 84, 179

Egan, M. P., Price, S. D., Moshir, M. M., Cohen, M., \& Tedesco, E. 1999, The Midcourse Space Experiment Point Source Catalog, version 1.2, Explanatory Guide, AFRL-VS-TR-1999-1522, Air Force Research Laboratory

Evans, N. J., \& Blair, G. N. 1981, ApJ, 246, 394

Fazio, G. G., Hora, J. L., Allen, L. E., et al. 2004a, ApJ, 154, 10

Fazio, G. G., Ashby , M. L. N., Barmby, P., et al. 2004b, ApJS, 154, 39

Felli, M., \& Panagia, N. 1981, A\&A, 102, 424

Felli, M., Palagi, F., \& Tofani, G. 1992, A\&A, 255, 293

Felli, M., Testi, L., Valdettaro, R., \& Wang, J.-J. 1997, A\&A, 320, 594

Felli, M., Massi, F., Navarrini, A., et al. 2004, A\&A, 420, 553

Fey, A. L., Gaume, R. A., Claussen, M. J., \& Vrba, F. J. 1995, ApJ, 453, 308

Furuya, R., Kitamura, Y., Wootten, H. A., Claussen, M. J., \& Kawabe, R. 2001, ApJ, 559, L143

Furuya, R., Kitamura, Y., Wootten, H. A., Claussen, M. J., \& Kawabe, R. 2003, ApJS, 144, 71

Garay, G., \& Rodriguez, L. F. 1990, ApJ, 362, 191

Gibb, A. G., Wyrowski, F., \& Mundy, L. G. 2004, ApJ, 616, 301

Gómez, Y., Rodríguez, L. F., \& Martí, J. 1995, ApJ, 453, 268

Harju, J., Lehtinen, K., Booth, R. S., \& Zinchenko, I. 1998, A\&AS, 132, 211

Haschick, A. D., Menten, K. M., \& Baan, W. A. 1990, ApJ, 354, 556

Heaton, B. D., Little, L. T., \& Bishop, I. S. 1989, A\&A, 213, 148

Henkel, C., Haschick, A. D., \& Güsten, R. 1986, A\&A, 165, 197

Indebetouw, R., Mathis, J. S., Babler, B. L., et al. 2005, ApJ, 619, 931

Israel, F. P., \& Felli, M. 1978, A\&A, 63, 325

Krassner, J., Pipher, J. L., Sharpless, S., \& Herter, T. 1982, A\&A, 109, 223

Kurtz, S., Cesaroni, R., Churchwell, E., Hofner, P., \& Walmsley, C. M. 2000, Hot Molecular Cores and the Earliest Phases of High-Mass Star Formation, in Protostars and Planets IV, ed. V. Mannings, A. Boss, \& S. S. Russell (Tucson: University of Arizona press), 299

Kurtz, S., Hofner, P., \& Álvarez, C. V. 2004, ApJS, 155, 149

Maxia, C., Testi, L., Cesaroni, R., \& Walmsley, C. M. 2001, A\&A, 371, 287

Mezger, P. G. 1978, A\&A, 70, 565

Moscadelli, L., Cesaroni, R., \& Rioja, M. J. 2000, A\&A, 360, 663

Moscadelli, L., Cesaroni, R., \& Rioja, M. J. 2005, A\&A, 438, 889

Nakano, M., \& Yoshida, S. 1986, PASJ, 38, 531

Palagi, F., Cesaroni, R., Comoretto, G., Felli, M., \& Natale, V. 1993, A\&AS, 101,153

Panagia, N. 1973, AJ, 78, 929

Panagia, N., \& Felli, M. 1975, A\&A, 39, 1

Reid, M. J., \& Ho, P. T. P. 1985, ApJ, 288, L17

Rodríguez, L. F. 1996, Rev. Mex. Astron. Astrophys., 4, 7

Rodríguez, L. F. 1997, Thermal Radio Jets. In Herbig-Haro Flows and the Birth of Low Mass Stars, ed. Bo Reipurth, \& C. Bertout (Dordrecht: Kluwer Academic Publishers), IAU Symp., 182, 83

Simon, M., Felli, M., Cassar, L., Fischer, J., \& Massi, M. 1983, ApJ, 266, 623

Tofani, G., Felli, M., Taylor, G. B., \& Hunter, T. R. 1995, A\&AS, 112, 299

Valdettaro, R., Palla, F., Brand, J., et al. 2002, A\&A, 383, 244

Watt, S., \& Mundy, L. G. 1999, ApJS, 125, 143

Whitney, B. A., Wood, K., Bjorkman, J. E., \& Cohen, M. 2003, ApJ, 598, 1079

Whitney, B. A., Indebetouw, R., Babler, B. L., et al. 2004, ApJS, 154, 315

Wood, D. O. S., \& Churchwell, E. 1989, ApJS, 69, 831 